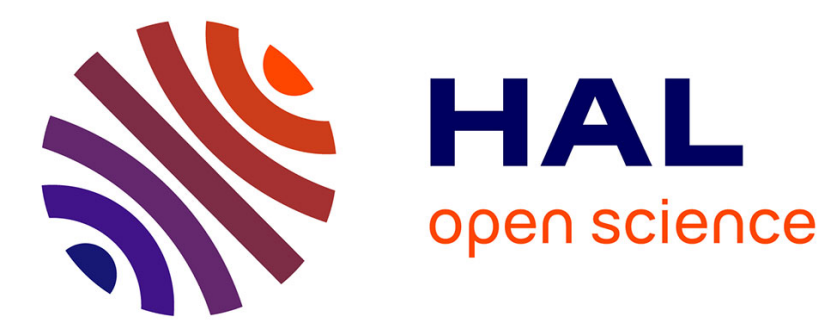

\title{
Local and global indeterminacy in two-sector models of endogenous growth
}

\author{
Paulo Brito, Alain Venditti
}

\section{To cite this version:}

Paulo Brito, Alain Venditti. Local and global indeterminacy in two-sector models of endogenous growth. 2009. halshs-00408018

\section{HAL Id: halshs-00408018 \\ https://shs.hal.science/halshs-00408018}

Preprint submitted on 28 Jul 2009

HAL is a multi-disciplinary open access archive for the deposit and dissemination of scientific research documents, whether they are published or not. The documents may come from teaching and research institutions in France or abroad, or from public or private research centers.
L'archive ouverte pluridisciplinaire HAL, est destinée au dépôt et à la diffusion de documents scientifiques de niveau recherche, publiés ou non, émanant des établissements d'enseignement et de recherche français ou étrangers, des laboratoires publics ou privés. 


\section{GREQAM}

Document de Travail

Groupement de Recherche en Economie Quantitative d'Aix-Marseille - UMR-CNRS 6579 Ecole des Hautes Etudes en Sciences Sociales Universités d'Aix-Marseille II et III

LOCAL AND GLOBAL INDETERMINACY IN TWO-SECTOR MODELS OF ENDOGENOUS GROWTH n²009-15

\section{Paulo BRITO Alain VENDITTI}

May 2009

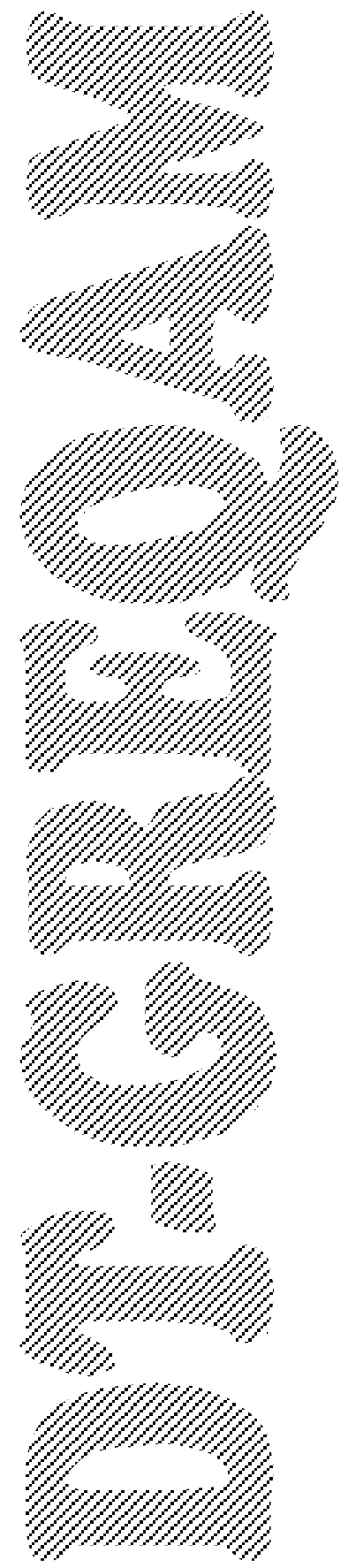




\title{
Local and global indeterminacy in two-sector models of endogenous growth*
}

\author{
Paulo BRITO \\ ISEG - Technical University of Lisbon and UECE, Lisbon, Portugal
E-mail: pbrito@iseg.utl.pt \\ and \\ Alain VENDITTI \\ CNRS - GREQAM, Marseille, France \\ E-mail: Alain.Venditti@univmed.fr \\ First version: September 2000; Revised: May 2009
}

\begin{abstract}
In this paper we consider a two-sector endogenous growth model where the productions of the final good and human capital require economy-wide external effects. Assuming constant returns to scale at the private and social levels, we show that local and global indeterminacy of equilibrium paths are compatible with any values for the elasticity of intertemporal substitution in consumption and any sign for the capital intensity difference across the two sectors.
\end{abstract}

Keywords: Two-sector model, endogenous growth, economy-wide externalities, local and global indeterminacy

Journal of Economic Literature Classification Numbers: C62, E32, O41.

${ }^{*}$ We thank E. Augeraud-Véron, J.P. Drugeon, C. García-Peñalosa, O. Licandro, P. Michel, K. Nishimura, M. Santos and P. Wang for useful comments and suggestions. This paper has also benefited from presentations at the conference "Les aspects intertemporels et intergénérationnels de la macroéconomie: Colloque en l'honneur de Philippe Michel", Paris, November 2002, the "8th SpiE Annual Meeting", Lisbon, June 2003, the "EUI-ECO Macroeconomics Research Workshop 2002-3", European University Institute, Florence, June 2003, and the workshop "Market imperfections and macroeconomic instability", Lille, March 2008. Financial support by Fundação para a Ciência e a Tecnologia, Portugal, and by French National Research Agency Grant (ANR-08-BLAN-0245-01) is gratefully acknowledged. This paper was part of project POCTI/eco/13028/98, and is part of the Multi-annual Funding Project POCI/U0436/2006. 


\section{Introduction}

This paper is concerned with the existence of multiple equilibria in twosector models of endogenous growth with economy-wide external effects $\grave{a}$ la Romer [28]. Most of the papers on multiple equilibria available in the literature provide a separate analysis of local versus global indeterminacy and give strong restrictions on technologies and/or preferences. Our aim is then to develop a two-sector formulation in which the existence of local and global indeterminacy can be analysed simultaneously and to provide less restrictive sufficient conditions than those usually available. Local indeterminacy is associated with the existence of a continuum of equilibrium paths from different initial conditions converging towards a given stationary balanced growth path, while global indeterminacy concerns the existence of multiple equilibrium paths from a given initial condition converging towards different stationary balanced growth paths.

The existence of local indeterminacy and multiple converging balanced growth paths have been a major concern of the literature dealing with twosector endogenous growth models. Depending on the assumptions on the externalities, different types of conclusions have been provided. Benhabib and Perli [7] and Xie [31] consider the Lucas [19] formulation with aggregate human capital externalities in the final good sector only. They prove the uniqueness of the stationary balanced growth path and show that the existence of local indeterminacy requires a large enough elasticity of intertemporal substitution in consumption. ${ }^{1}$ Benhabib et al. [6], Mino [21] and Nishimura and Venditti [25] consider sector-specific externalities in both sectors. Uniqueness of the stationary balanced growth path is also obtained and local indeterminacy arises under conditions on the capital intensity difference across sectors, namely if the final good sector is more physical capital intensive from the social perspective but more human capital intensive from the private perspective than the human capital sector. ${ }^{2}$ It is worth noting

\footnotetext{
${ }^{1}$ See also Mitra [22] for a similar analysis of a discrete-time version of the Lucas model.

${ }^{2}$ Similar results are also obtained by Bond et al. [10] in a model with taxes instead of externalities, by Raurich [26] in a model with taxes and government spending, and by Ben-Gad [5] in a model with both taxes and sector-specific externalities.
} 
that no particular condition on the elasticity of intertemporal substitution in consumption is necessary in this case.

Concerning the existence of multiple stationary balanced growth paths, an augmented version of the Lucas model is usually considered. In a first set of contributions we find Benhabib and Perli [7] and Ladrón-de-Guevara et al. $[16,17]$ who introduce endogenous leisure to obtain two stationary solutions. In a second and more recent set of contributions, García-Belenguer [15] and Mattana et al. [20] focus on different specifications for the external effects. García-Belenguer [15] shows the existence of multiple stationary balanced growth paths with a Lucas model augmented to include aggregate physical capital externalities in the production of the final good and decreasing returns to scale in the human capital accumulation process. Local indeterminacy is also obtained but still requires a large enough elasticity of intertemporal substitution in consumption. ${ }^{3}$ In Mattana et al. [20], multiple stationary balanced growth paths are derived from the introduction of externalities in the human capital sector, and global indeterminacy is exhibited through an homoclinic bifurcation.

In this paper, we develop a formulation which allows to provide a joint analysis of multiple stationary balanced growth paths and multiple competitive equilibrium balanced growth paths. Building on the Lucas [19] framework generalized by Mulligan and Sala-i-Martin [23], we consider a two-sector endogenous growth model where the productions of the final good (used for consumption and as physical capital) and human capital require economy-wide external effects. Our formulation differs from that of Lucas [19] in two dimensions: First the technology for the production of human capital depends on both physical and human capital, ${ }^{4}$ and is also affected by the externalities. Second, the economy-wide external effects are defined as physical capital by unit of efficient labor. In other words, the externalities are formulated in such a way that the returns to scale in both sectors are

\footnotetext{
${ }^{3}$ Similar results have been obtained by Drugeon et al. [14] and Nishimura et al. [24] in a two-sector endogenous growth model with a pure consumption good and an investment good, and economy-wide capital externalities.

${ }^{4}$ See also Rebelo [27].
} 
constant at the private and social levels. ${ }^{5}$ From this point of view, it allows to avoid to refer to the existence of positive profit at the private level as in Benhabib et al. [6], Mino [21], or Nishimura and Venditti [25]. Such a formulation is also embedded in the general model considered by Mulligan and Sala-i-Martin [23] but has never been analyzed in the literature. ${ }^{6}$

Our main results are the following. First we give simple conditions for the existence of one or two stationary balanced growth paths. Second, we show that for a given stationary solution, two kinds of local indeterminacy can occur: a local indeterminacy of order 2 in which the stable manifold has dimension 2, and a local indeterminacy of order 3 in which the stable manifold has dimension 3. This last case is particularly interesting as we prove that in a configuration with two stationary solutions, one can be locally indeterminate of order 2 while the other is locally indeterminate of order 3. This type of generalized local and global indeterminacy has never been illustrated previously.

We actually provide improved sufficient conditions for local and global indeterminacy with respect to the literature. We show indeed that the existence of multiple stationary solutions and the existence of a continuum of equilibrium paths are compatible with both types of physical capital intensity differences across sectors at the private level and low values for the elasticity of intertemporal substitution in consumption. In particular these results can be obtained with a standard logarythmic utility function in which the elasticity of intertemporal substitution in consumption is unitary provided the final good sector is human capital intensive at the private level. Note however that in this case, only a local indeterminacy of order 2 can occur and requires the existence of two stationary balanced growth paths. In other words, local and global indeterminacy are closely related.

When a non unitary elasticity of intertemporal substitution in consumption is considered, we prove in particular that a local indeterminacy of order 3 can arise in two configurations: either when the final good is more inten-

\footnotetext{
${ }^{5}$ Such an assumption meets the findings of Basu and Fernald [4] according to which the aggregate returns to scale in the US are roughly constant.

${ }^{6}$ Drugeon [13] considers a similar formulation but with sector-specific externalities.
} 
sive in human capital at the private level and the elasticity of intertemporal substitution in consumption is lower than unity, or when the final good is more intensive in physical capital at the private level and the elasticity of intertemporal substitution in consumption is larger than unity.

To summarize, we show that the existence of multiple equilibrium balanced growth paths can arise under a wide variety of parameters configurations, i.e. for any sign of the capital intensity difference at the private level, and any value of the elasticity of intertemporal substitution in consumption. These results differ from most of the previous analysis of models with externalities where indeterminacy has been shown to arise only for large intertemporal substitutability. ${ }^{7}$ A second contribution of our paper is that we can place it in the context of the literature on poverty traps. ${ }^{8}$ We prove that global indeterminacy is always associated with the existence of a poverty trap when the elasticity of intertemporal substitution in consumption is larger than unity, while it can be characterized by a high growth balanced growth path which has a higher dimension of stability than the low growth one when the elasticity of intertemporal substitution is lower than unity. In this last case, the poverty trap can be avoided by correctly choosing the equilibrium path.

The rest of the paper is organized as follows: Section 2 presents the model and solves the competitive equilibrium. The existence and multiplicity of stationary balanced growth paths are studied in Section 3 and the characteristic polynomial associated with the linearized dynamical system is computed. Section 4 contains the analysis of local and global indeterminacy. Section 5 provides concluding comments. The proofs are in the Appendix.

\section{The model}

We consider the model formulated by Mulligan and Sala-i-Martin [23] with constant point-in-time returns and different technologies. The representative household (-firm owner) decides optimally over consumption streams and

\footnotetext{
${ }^{7}$ See Benhabib and Perli [7], García-Belenguer [15].

${ }^{8}$ See Azariadis and Stachurski [2].
} 
allocations of physical $\left(K_{1}\right)$ and human $\left(K_{2}\right)$ capital between manufacturing and educational activities, by solving the intertemporal problem:

$$
\begin{array}{cl}
\max _{C(t), K_{11}(t), K_{21}(t), K_{12}(t), K_{22}(t)} & \int_{0}^{+\infty} \frac{C(t)^{1-\sigma}-1}{1-\sigma} e^{-\rho t} d t \\
\text { s.t. } & \dot{K}_{1}(t)=Y_{1}(t)-\delta_{1} K_{1}(t)-C(t) \\
& \dot{K}_{2}(t)=Y_{2}(t)-\delta_{2} K_{2}(t) \\
& Y_{j}(t)=e_{j}(t) K_{1 j}(t)^{\beta_{1 j}} K_{2 j}(t)^{\beta_{2 j}}, j=1,2 \\
& K_{i}(t)=K_{i 1}(t)+K_{i 2}(t), i=1,2 \\
& K_{j}(0),\left\{e_{j}(t)\right\}_{t=0}^{+\infty}, j=1,2, \text { given }
\end{array}
$$

where $\sigma>0$ is the inverse of the elasticity of intertemporal substitution in consumption, $\rho>0$ is the discount factor, $K_{i j}$ is the amount of capital good $i$ used in sector $j$ and $\delta_{i}$ is the rate of depreciation of capital $K_{i}, i=1,2$. In order to simplify the analysis, we assume that the depreciation rate of both physical and human capital is equal to zero, i.e. $\delta_{1}=\delta_{2}=0$. Of course, all our results would be preserved by continuity if we introduce small enough depreciation rates for both stocks.

Each technology is homogeneous of degree one at the private level, i.e. $\sum_{i=1}^{2} \beta_{i j}=1, j=1,2$, and contains some Romer-type productive externalities. We assume that these externalities are given as functions of physical capital by unit of efficient labor, namely

$$
e_{j}(t)=\bar{k}(t)^{b_{j}}, j=1,2
$$

with $\bar{k}(t)=\bar{K}_{1}(t) / \bar{K}_{2}(t), \bar{K}_{1}(t)$ and $\bar{K}_{2}(t)$ the average stocks of physical and human capital in the economy, and $b_{j} \in[0,1]$. At the equilibrium, we have $\bar{K}_{i}=K_{i}, \bar{k}=k=K_{1} / K_{2}$ and the technologies at the social level are

$$
\begin{aligned}
& Y_{1}(t)=K_{11}(t)^{\beta_{11}} K_{21}(t)^{\beta_{21}} k(t)^{b_{1}} \\
& Y_{2}(t)=K_{12}(t)^{\beta_{12}} K_{22}(t)^{\beta_{22}} k(t)^{b_{2}}
\end{aligned}
$$

They are both homogeneous of degree 1 at the private and social levels.

Let us denote the matrix of private Cobb-Douglas coefficients as follows

$$
B=\left(\begin{array}{ll}
\beta_{11} & \beta_{12} \\
\beta_{21} & \beta_{22}
\end{array}\right)
$$

with $\beta_{21}=1-\beta_{11}$ and $\beta_{22}=1-\beta_{12}$. 
Assumption 1. The technological coefficients at the private level satisfy $0<\beta_{i j}<1$ for $i, j=1,2$ and $\beta_{11}-\beta_{12} \neq 0$.

The inverse of $B$, denoted by $\Psi$, is thus

$$
\Psi=\left(\begin{array}{ll}
\psi_{11} & \psi_{12} \\
\psi_{21} & \psi_{22}
\end{array}\right)=\frac{1}{\beta_{11}-\beta_{12}}\left(\begin{array}{cc}
\beta_{22} & -\beta_{12} \\
-\beta_{21} & \beta_{11}
\end{array}\right),
$$

The Hamiltonian and Lagrangian in current value are

$$
\begin{aligned}
\mathcal{H} & =\frac{C^{1-\sigma}-1}{1-\sigma}+P_{1}\left(Y_{1}-C\right)+P_{2} Y_{2} \\
\mathcal{L} & =\mathcal{H}+R_{1}\left(K_{1}-K_{11}-K_{12}\right)+R_{2}\left(K_{2}-K_{21}-K_{22}\right)
\end{aligned}
$$

where $P_{i}$ is the utility price and $R_{i}$ the rental rate of good $i=1,2$. The Pontryagin maximum principle gives necessary conditions for a maximum:

$$
\begin{aligned}
C^{-\sigma} & =P_{1} \\
K_{i j} & =P_{j} \frac{\beta_{i j}}{R_{i}} Y_{j}, \text { for } i, j=1,2 \\
K_{i} & =K_{i 1}+K_{i 2}, \text { for } i=1,2 \\
\dot{P}_{i} & =\rho P_{i}-R_{i}, \text { for } i=1,2 \\
\dot{K}_{i} & =Y_{i}-C \iota_{1}, \text { for } i=1,2
\end{aligned}
$$

where $\iota_{1}:=\{1$ if $i=1, \quad 0$ if $i=2\}$. From the first-order condition (3) and (4), we can easily check that the maximised Hamiltonian is concave in $\left(K_{1}, K_{2}\right)$ and satisfies the Arrow's condition. ${ }^{9}$ It follows that any path that satisfies the conditions (3)-(7) together with the transversality conditions

$$
\lim _{t \rightarrow+\infty} P_{1}(t) K_{1}(t) e^{-\rho t}=\lim _{t \rightarrow+\infty} P_{2}(t) K_{2}(t) e^{-\rho t}=0
$$

for any given path of external effects $\left\{e_{1}(t), e_{2}(t)\right\}_{t=0}^{+\infty}$ and initial conditions $\left(K_{1}(0), K_{2}(0)\right)$, is an optimal solution of problem (1).

Lemma 1. Solving the first order conditions (4)-(5) gives the rental rates

$$
R_{i}=\left(P_{1} e_{1} \beta_{1}^{*}\right)^{\psi_{1 i}}\left(P_{2} e_{2} \beta_{2}^{*}\right)^{\psi_{2 i}}, \quad i=1,2
$$

where

$$
\beta_{j}^{*}=\beta_{1 j}^{\beta_{1 j}} \beta_{2 j}^{\beta_{2 j}}, \quad j=1,2
$$

and the optimal production levels

$$
Y_{j}=e_{j}\left(\psi_{1 j} R_{1} K_{1}+\psi_{2 j} R_{2} K_{2}\right)\left(\beta_{1 j} / R_{1}\right)^{\beta_{1 j}}\left(\beta_{2 j} / R_{2}\right)^{\beta_{2 j}}, \quad i=1,2 .
$$

Proof. See Appendix 6.1.

\footnotetext{
${ }^{9}$ See Leonard and Van Long [18].
} 
Remark: Private constant returns imply that the rental rates are homogeneous of degree one in the prices, since $\psi_{11}+\psi_{21}=\psi_{12}+\psi_{22}=1$.

Consider now the formulation of external effects (2). After substitution of this expression into the rental rates (8), and from the first order conditions (6)-(7), we get the following result:

Proposition 1. Under Assumption 1, denote $A^{-1}=\left[\alpha_{i j}\right]$ with $\alpha_{i j}=$ $R_{j} \psi_{i j} / P_{i}, r_{i}=R_{i} / P_{i}, P=\left(P_{1}, P_{2}\right)$ and $K=\left(K_{1}, K_{2}\right)$. The equilibrium paths are solution of the following dynamical system

$$
\begin{aligned}
\dot{P}_{i} & =P_{i}\left(\rho-r_{i}(P, K)\right), \text { for } i=1,2 \\
\dot{K}_{i} & =\alpha_{i 1} K_{1}+\alpha_{i 2} K_{2}-C \iota_{1}, \text { for } i=1,2 .
\end{aligned}
$$

with

$$
\begin{aligned}
C & =C\left(P_{1}\right)=P_{1}^{-1 / \sigma} \\
R_{i}(P, K) & =\left(\beta_{1}^{*}\right)^{\psi_{1 i}}\left(\beta_{2}^{*}\right)^{\psi_{2 i}} P_{1}^{\psi_{1 i}} P_{2}^{\psi_{2 i}}\left(K_{1} / K_{2}\right)^{b_{1} \psi_{1 i}+b_{2} \psi_{2 i}} \quad i=1,2,
\end{aligned}
$$

Remark: Denoting $\pi=P_{2} / P_{1}$ and $k=K_{1} / K_{2}$, the rental rates $r_{i}=R_{i} / P_{i}$ become

$$
r_{i}(\pi, k)=\left(\beta_{1}^{*}\right)^{\psi_{1 i}}\left(\beta_{2}^{*}\right)^{\psi_{2 i}} \pi^{\psi_{2 i}} k^{b_{1} \psi_{1 i}+b_{2} \psi_{2 i}}
$$

Note that $r_{i}(\pi, k)$ is a function of $k$ only if there are externalities. If there are no externalities, it only depends on prices.

We now define a balanced growth path, BGP, as the state where all the variables grow at a constant rate. We thus rule out paths with ever increasing growth rates which will not satisfy the transversality conditions. As the social production functions are homogeneous of degree 1 at the private and social levels, along a BGP physical and human capital will grow at the same rate denoted $\gamma$. It follows that along a BGP the prices $P_{1}$ and $P_{2}$ of physical and human capital will also grow at the same rate denoted $\gamma_{p}$. From the dynamical equation characterizing the behavior of physical capital, the stationary balanced growth rate of consumption will be also equal to that of physical capital, i.e. $\gamma$. Using the normalization of variables as introduced by Caballe and Santos [11], we then define a BGP as follows: $K_{1}(t)=k_{1}(t) e^{\gamma t}, K_{2}(t)=k_{2}(t) e^{\gamma t}, C(t)=c(t) e^{\gamma t}, P_{1}(t)=p_{1}(t) e^{\gamma_{p} t}$ and 
$P_{2}(t)=p_{2}(t) e^{\gamma_{p} t}$, for all $t \geq 0$, with $k_{1}(t), k_{2}(t), c(t), p_{1}(t)$ and $p_{2}(t)$ the stationarised values for $K_{1}(t), K_{2}(t), C(t), P_{1}(t)$ and $P_{2}(t)$. Substituting these new variables into the dynamical system (9) gives $\gamma_{p}=-\sigma \gamma$. As usual along a BGP prices decrease at a constant rate which is equal to the growth rate of physical capital divided by the elasticity of intertemporal substitution in consumption.

\section{Endogenous growth: existence and multiplicity of stationary BGP and local dynamics}

The stationary balanced growth rates and the levels for the stationarised variables consistent with the BGP, $\bar{c}, \bar{k}_{1}, \bar{k}_{2} \bar{p}_{1}, \bar{p}_{2}$ and $\bar{\pi}=\bar{p}_{2} / \bar{p}_{1}$, are derived from the equilibrium set of the dynamical system (9) which becomes

$$
\begin{aligned}
& \dot{p}_{1}=p_{1}\left(\rho+\sigma \gamma-r_{1}(\pi, k)\right) \\
& \dot{p}_{2}=p_{2}\left(\rho+\sigma \gamma-r_{2}(\pi, k)\right) \\
& \dot{k}_{1}=\left(\alpha_{11}-\gamma\right) k_{1}+\alpha_{12} k_{2}-p_{1}^{-1 / \sigma} \\
& \dot{k}_{2}=\alpha_{21} k_{1}+\alpha_{22} k_{2}-\gamma k_{2} .
\end{aligned}
$$

and the transversality conditions are now stated as follows:

$$
\lim _{t \rightarrow+\infty} p_{1}(t) k_{1}(t) e^{[\gamma(1-\sigma)-\rho] t}=\lim _{t \rightarrow+\infty} p_{2}(t) k_{2}(t) e^{[\gamma(1-\sigma)-\rho] t}=0
$$

We introduce the following restriction in order to satisfy these boundary conditions:

Assumption 2. $\rho-\gamma(1-\sigma)>0$

\subsection{Stationary balanced growth path}

The equilibrium point of the system (11)-(14) determines the stationary BGP. It is an at-least one dimensional manifold in $\left(\gamma, p_{1}, p_{2}, k_{1}, k_{2}\right)$. Using (11) and (12), we get $r_{1}(\pi, k)=r_{2}(\pi, k)=r(\pi, k)$ and

$$
\gamma=\frac{r(\pi, k)-\rho}{\sigma}
$$

where $0<\gamma<r$ from the transversality conditions. 
Lemma 2. A stationary balanced growth rate $\bar{\gamma}=(\bar{r}-\rho) / \sigma$ is obtained from a stationary rental rate $\bar{r}$ which is a solution of

$$
\mathcal{F}(r)=\mathcal{G}(r)
$$

with $\mathcal{F}(r)=\sigma \beta_{21} \tilde{\beta} r^{\mu}, \mathcal{G}(r)=\left[\beta_{11}(\sigma-1)+\beta_{12}\right] r+\rho\left(\beta_{11}-\beta_{12}\right)$ and

$$
\tilde{\beta}=\left(\left(\beta_{1}^{*}\right)^{\beta_{12}+b_{2}}\left(\beta_{2}^{*}\right)^{\beta_{21}-b_{1}}\right)^{-1 /\left(\beta_{12} b_{1}+\beta_{21} b_{2}\right)}, \quad \mu=1+\frac{\beta_{12}+\beta_{21}+b_{2}-b_{1}}{\beta_{12} b_{1}+\beta_{21} b_{2}}
$$

Proof. See Appendix 6.2.

Let us define the following values:

$$
r^{*}=\left(\frac{\beta_{11}(\sigma-1)+\beta_{12}}{\mu \sigma \beta_{21} \tilde{\beta}}\right)^{\frac{1}{\mu-1}}, \quad \rho^{*}=\frac{(1-\mu)\left[\beta_{11}(\sigma-1)+\beta_{12}\right]}{\mu\left(\beta_{11}-\beta_{12}\right)} r^{*}
$$

When $\rho=\rho^{*}, r^{*}$ is a tangency point between $\mathcal{F}(r)$ and $\mathcal{G}(r)$. Consider also

$$
\rho_{1}=(1-\sigma)\left(\frac{\beta_{21}}{\beta_{12}} \tilde{\beta}\right)^{1 /(1-\mu)}, \quad \rho_{2}=\left(\frac{\beta_{21}}{\beta_{11}} \tilde{\beta}\right)^{1 /(1-\mu)}
$$

Note that as $\rho_{1}$ can be negative and the difference $\rho_{2}-\rho_{1}$ can be positive or negative, we will denote $\rho_{1}^{*}=\max \left\{0, \min \left\{\rho_{1}, \rho_{2}\right\}\right\}$ and $\rho_{2}^{*}=\max \left\{\rho_{1}, \rho_{2}\right\}$. Note also that if $\sigma \geq 1, \rho_{1}^{*}=0$ and $\rho_{2}^{*}=\rho_{2}$. Let us finally introduce

$$
\mu_{1}=\frac{\beta_{11}(\sigma-1)+\beta_{12}}{\sigma \beta_{11}}, \quad \mu_{2}=\frac{\beta_{11}(\sigma-1)+\beta_{12}}{\sigma \beta_{12}}
$$

From all this, we now study the existence of a stationary balanced growth rate. We first provide conditions for multiplicity.

Theorem 1. Let $\hat{\sigma}=\left(\beta_{11}-\beta_{12}\right) / \beta_{11}$. Under Assumptions 1-2, there exist two stationary balanced growth rates $0<\bar{\gamma}_{1}<\bar{\gamma}_{2}$ if and only if one of the following sets of conditons holds:

i) $\beta_{11}<\beta_{12}, \mu \in\left(\mu_{2}, \mu_{1}\right) \subset(1,+\infty)$ and $\rho \in\left(\rho_{2}^{*}, \rho^{*}\right)$;

ii) $\beta_{11}>\beta_{12}, \sigma \geq \hat{\sigma}, \mu \in\left(\mu_{1}, \mu_{2}\right) \subset(0,1)$ and $\rho \in\left(\rho_{2}^{*}, \rho^{*}\right)$;

iii) $\beta_{11}>\beta_{12}, \sigma \in(0, \hat{\sigma}], \mu \in\left(\mu_{2}, \mu_{1}\right) \subset(-\infty, 0)$ and $\rho \in\left(\rho^{*}, \rho_{1}^{*}\right)$.

Proof. See Appendix 6.3.

Theorem 1 shows that multiple stationary balanced growth rates arise in both configurations for the capital intensity difference at the private level. Note however that if the amount of externalities is the same in both sectors, i.e. $b_{1}=b_{2}=b>0$, or if there is no externality in the final good sector, 
i.e. $b_{1}=0$, or if the Lucas [19] formulation for the accumulation law of the human capital sector is assumed, i.e. $b_{2}=\beta_{22}=0$, cases ii) and iii) cannot occur. However, multiple stationary BGPs are still a possible outcome through case i) as shown in the following corollary:

Corollary 1. Under Assumptions 1-2, there exist two stationary balanced growth rates $0<\bar{\gamma}_{1}<\bar{\gamma}_{2}$ if one of the following sets of conditions holds:

i) $b_{1}=b_{2}=b>0, \beta_{11}<\beta_{12}, \mu \in\left(\mu_{2}, \mu_{1}\right) \subset(1,+\infty)$ and $\rho \in\left(\rho_{2}^{*}, \rho^{*}\right)$;

ii) $b_{1}=0, b_{2}>0, \beta_{11}<\beta_{12}, \mu \in\left(\mu_{2}, \mu_{1}\right) \subset(1,+\infty)$ and $\rho \in\left(\rho_{2}^{*}, \rho^{*}\right)$;

iii) $b_{1}>0, b_{2}=\beta_{22}=0, \mu \in\left(\mu_{2}, \mu_{1}\right) \subset(1,+\infty)$ and $\rho \in\left(\rho_{2}^{*}, \rho^{*}\right)$.

Proof. See Appendix 6.4.

On the one hand, Corollary 1-ii) shows that the existence of multiple stationary BGPs does not require externalities in the final good sector provided there are external effects in the human capital sector. This conclusion is similar to the one obtained by Mattana et al. [20]. ${ }^{10}$ On the other hand, Corollary 1-iii) proves that multiple stationary BGPs also arise if the accumulation of human capital does not depend on physical capital and externalities provided there are external effects in the final good sector. This conclusion is similar to the one obtained by García-Belenguer [15].

Simple conditions for the existence and uniqueness of a stationary balanced growth rate are now stated in the following Theorem. ${ }^{11}$

Theorem 2. Under Assumptions 1-2, there exists a unique stationary balanced growth rate $\bar{\gamma}>0$ if and only if one of the following sets of conditons holds:

i) $\sigma \geq 1$ and $\rho<\rho_{2}^{*}=\rho_{2}$;

ii) $\sigma \in(0,1)$, and $\rho \in\left(\rho_{1}^{*}, \rho_{2}^{*}\right)$.

Proof. See Appendix 6.5.

\footnotetext{
${ }^{10}$ See also Chamley [12].

${ }^{11}$ Without externalities, i.e. if $b_{1}=b_{2}=0, r=\left[\left(\beta_{1}^{*}\right)^{\beta_{12}}\left(\beta_{2}^{*}\right)^{\beta_{21}}\right]^{\frac{1}{\beta_{12}+\beta_{21}}}$ is the unique stationary value for the rental rate of capital so that there exists a unique stationary BGP (see d'Autume and Michel [1], Caballe and Santos [11], Rebelo [27]).
} 
Theorems 1 and 2 can be summarized by the following Table:

\begin{tabular}{|c|c|c|c|c|c|}
\hline & $\mu<0$ & $\mu=0$ & $\mu \in(0,1)$ & $\mu=1$ & $\mu>1$ \\
\hline$\gamma_{\beta_{11}<\beta_{1}}$ & 1 & 1 & 1 & 1 & 1 or 2 \\
\hline$\beta_{11}>\beta_{12}, \sigma>\hat{\sigma}$ & 1 & 1 & 1 or 2 & 1 & 1 \\
\hline${ }_{\beta_{11}>\beta_{12}, \sigma}=\hat{\sigma}$ & 1 & 0 & 1 & 1 & 1 \\
\hline$\varlimsup_{\beta_{11}>\beta_{12}, \sigma \in[0, \hat{\sigma})}$ & 1 or 2 & 1 & 1 & 1 & 1 \\
\hline
\end{tabular}

Table 1: Existence of stationary BGP: uniqueness vs. multiplicity

In order to clarify the implications of our results, we provide in Figures 1 and 2 geometrical illustrations of some cases covered in Theorems 1 and 2 .

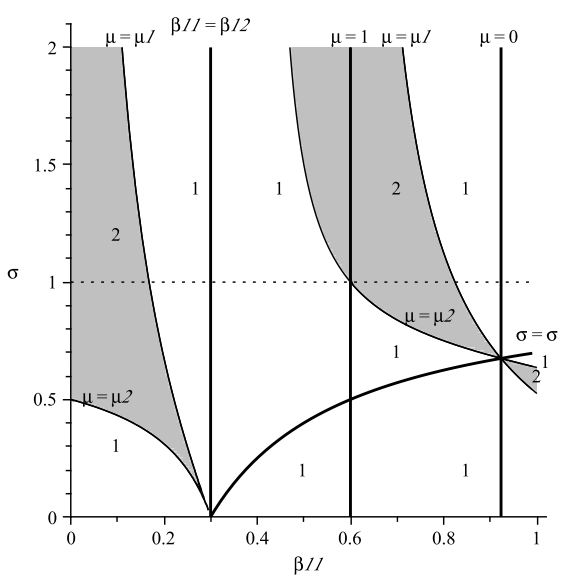

Figure 1: $1>b_{1}-b_{2}>0$. 


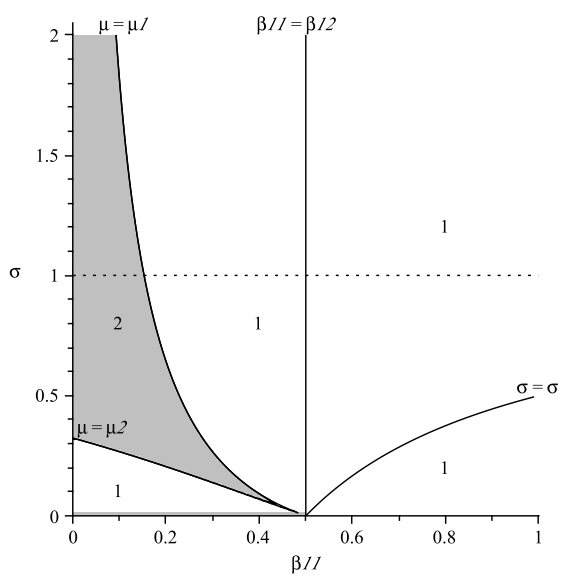

Figure 2: $b_{1}-b_{2}<0$.

\subsection{Local dynamics}

Linearizing the dynamical system (11)-(14) around a stationary BGP gives the characteristic polynomial.

Proposition 2. Under Assumptions 1-2, let $\bar{r}$ be a solution of equation (17), $\bar{\gamma}=(\bar{r}-\rho) / \sigma$ and $\bar{\delta}=\bar{r}-\bar{\gamma}>0$. Consider also $\mathcal{G}(r)$ and $\mu$ respectively defined by equations (17) and (18). The characteristic polynomial is

$$
g(\lambda)=\lambda^{4}-\mathcal{T}(\bar{r}) \lambda^{3}+\mathcal{S}(\bar{r}) \lambda^{2}-\mathcal{D}(\bar{r}) \lambda+\Sigma(\bar{r})=0
$$

with $\Sigma(\bar{r})=0$,

$$
\begin{aligned}
\mathcal{D}(\bar{r})= & -\bar{\delta} \frac{\bar{r}^{1-\mu}\left[\bar{r}-\bar{\gamma}\left(\beta_{11}-\beta_{12}\right)\right]\left(\beta_{12} b_{1}+\beta_{21} b_{2}\right)\left[\mathcal{G}(\bar{r})(\mu-1)+\rho\left(\beta_{11}-\beta_{12}\right)\right]}{\left(\beta_{11}-\beta_{12}\right)^{2} \sigma \beta_{21} \tilde{\beta}} \equiv \bar{\delta} \tilde{\mathcal{D}}(\bar{r}) \\
\mathcal{T}(\bar{r})= & 2 \bar{\delta}-\bar{r} \frac{\left(b_{2}-b_{1}\right)}{\left(\beta_{11}-\beta_{12}\right)^{2}}+\frac{\bar{\delta}\left[\bar{\gamma}\left(\beta_{12} b_{1}+\beta_{21} b_{2}\right)+\bar{\delta} b_{1}\right]}{\beta_{21} \tilde{\bar{r}} \bar{r} \mu} \equiv 2 \bar{\delta}+\tilde{\mathcal{T}}(\bar{r}) \\
\mathcal{S}(\bar{r})= & \bar{\delta}(\mathcal{T}(\bar{r})-\bar{\delta})+\bar{\delta}^{-1} \mathcal{D}(\bar{r})+\mathcal{Y}(\bar{r}) \equiv \bar{\delta}(\tilde{\mathcal{T}}(\bar{r})+\bar{\delta})+\tilde{\mathcal{D}}(\bar{r})+\mathcal{Y}(\bar{r}) \\
& \mathcal{Y}(\bar{r})=\bar{r}^{1-\mu}\left(\frac{1-\sigma}{\sigma}\right) \frac{\left[\bar{r}-\bar{\gamma}\left(\beta_{11}-\beta_{12}\right)\right]\left[\bar{\gamma}\left(\beta_{12} b_{1}+\beta_{21} b_{2}\right)+\bar{\delta} b_{1}\right]}{\left(\beta_{11}-\beta_{12}\right) \beta_{21} \tilde{\beta}}
\end{aligned}
$$

and

Proof. See Appendix 6.6.

As $\Sigma(\bar{r})=0$, one eigenvalue, say $\lambda_{4}=0$, is equal to zero. This property comes from the fact that we consider the dynamical system (11)-(14) which has been stationarised. Then, we get $\lambda_{1}+\lambda_{2}+\lambda_{3}=\mathcal{T}(\bar{r}), \lambda_{1} \lambda_{2}+\lambda_{1} \lambda_{3}+$ $\lambda_{2} \lambda_{3}=\mathcal{S}(\bar{r})$ and $\lambda_{1} \lambda_{2} \lambda_{3}=\mathcal{D}(\bar{r})$. 
In the current economy, there are two capital goods whose initial values are given. Any solution of the dynamical system (11)-(14) that converges to a stationary BGP $\bar{\gamma}$, i.e. to the associated steady state $\left(\bar{k}_{1}, \bar{k}_{2}, \bar{p}_{1}, \bar{p}_{2}\right) \equiv$ $(\bar{k}, \bar{p})$, and that satisfies the transversality conditions (15) is an equilibrium path. Therefore, given $\left(k_{1}(0), k_{2}(0)\right)$, if there is more than one set of initial prices $\left(p_{1}(0), p_{2}(0)\right)$ in the stable manifold of the steady state under consideration, the equilibrium path from $\left(k_{1}(0), k_{2}(0)\right)$ will not be unique. Since one root is always equal to zero, if the Jacobian matrix has at least two roots with negative real parts, there will be a continuum of converging paths and thus a continuum of equilibria. The stationary BGP $\bar{\gamma}$, or the steady state $(\bar{k}, \bar{p})$, is then said to be locally indeterminate. In this case, we distinguish two kinds of local indeterminacy:

- a local indeterminacy of order 2 in which the stable manifold is two dimensional. All the converging equilibrium are then obtained from a projection of the three dimensional dynamical system defined by equations (11)(14) on the two dimensional subspace corresponding to the stable manifold.

- a local indeterminacy of order 3 in which the stable manifold is three dimensional. All the converging equilibrium are then directly obtained in the original three dimensional dynamical system.

Such a distinction has important consequences. We know indeed since Woodford [30] that local indeterminacy is a sufficient condition for the existence of sunspot equilibria. However, this conclusion relies on the assumption that all the characteristic roots are stable. Within such a framework, Woodford is able to exploit the (local) linear approximation of the dynamical system to prove his result. The problem comes from the fact that when there exist unstable roots, the consideration of the linear approximation does not provide correct conclusions. Indeed, it has been proved by Bloise [8] that when non-linearities are taken into account, sunspot equilibria cannot be constructed on the local stable manifold. Bloise then shows that the existence of sunspot equilibria on a full dimensional set can be obtained but requires more restrictive assumptions. ${ }^{12}$

\footnotetext{
${ }^{12}$ See also Bloise and Magris [9] for some application of this result to a cash-in-advance economy with capital accumulation.
} 
Definition 1. If the locally stable manifold of the stationary BGP $\bar{\gamma}$ has dimension $n$ greater than 1 , then $\bar{\gamma}$ is said to be locally indeterminate. More precisely, we will say that $\bar{\gamma}$ is locally indeterminate of order 2 if $n=2$ or locally indeterminate of order 3 if $n=3$.

If a stationary $\mathrm{BGP} \bar{\gamma}$ is not locally indeterminate then we call it locally determinate. This terminology will cover two different configurations: saddle-point stability in which there exists one unique converging equilibrium path or local instability. In this latter case either there exists some equilibrium path converging toward some periodic cycle or the externalities are such that there does not exist any equilibrium path. ${ }^{13}$

Beside the occurrence of local indeterminacy, as multiple BGPs can exist, global indeterminacy is also a possible outcome of our model. When two distinct BGPs $\bar{\gamma}_{1}$ and $\bar{\gamma}_{2}$ exist, from a given initial condition for physical and human capital, two distinct equilibrium paths converging towards different long-run positions can indeed coexist. The following definition provides a precise characterization for this configuration.

Definition 2. Consider an equilibrium path $\left(k_{1}^{*}(t), k_{2}^{*}(t), p_{1}^{*}(t), p_{2}^{*}(t)\right)$ converging to a BGP $\bar{\gamma}_{1}$ from a given initial condition $\left(k_{1}(0), k_{2}(0)\right)$. Global indeterminacy emerges if there exists another equilibrium path $\left(k_{1}(t), k_{2}(t), p_{1}(t), p_{2}(t)\right)$ starting from the same initial condition but converging to a different $B G P \bar{\gamma}_{1} \neq \bar{\gamma}_{2}$.

Global indeterminacy can emerge in various configurations:

i) when the two BGPs are saddle-point stable, ${ }^{14}$ or

ii) when one BGP is saddle-point stable while the other is locally indeterminate of order 2 or 3 , or

iii) when one BGP is locally indeterminate of order 2 while the other is locally indeterminate of order 3.

\footnotetext{
${ }^{13}$ See Santos [29].

${ }^{14}$ We cannot get two saddle point stable stationary BGPs. Such a configuration can occur if the equilibrium is given by singular ordinary differential equations, or differential algebraic equations with singularities, in which, infinite eigenvalues may exist.
} 
Local and global indeterminacy are then closely related. We will be particularly interested in the third configuration as there does not exist in the literature any illustration characterized by such a strong indeterminacy property.

\section{Local and global indeterminacy of BGP}

In order to simplify notations, a stationary balanced growth equilibrium will be defined in the rest of the paper by a pair $(\bar{r}, \bar{\gamma})$ such that $\mathcal{F}(\bar{r})=\mathcal{G}(\bar{r})$ and $\bar{\gamma}=(\bar{r}-\rho) / \sigma$. Our aim is to study the local stability properties of the stationary balanced growth rate exhibited in Section 3.1. The main difficulty comes from the fact that multiple BGPs can co-exist.

As a benchmark case, consider the formulation without external effects, i.e. $b_{1}=b_{2}=0$, which leads to an optimal growth model. Of course local and global indeterminacy are ruled out. Endogenous growth occurs however as there exists one unique stationary balanced growth rate provided the discount factor $\rho$ is small enough (see footnote 11 and Theorem 2). The characteristic polynomial becomes

$g(\lambda)=(\lambda-\bar{\delta})\left(\lambda^{2}-\bar{\delta} \lambda+\tilde{\mathcal{D}}_{0}(\bar{r})\right)=0$ with $\tilde{\mathcal{D}}_{0}(\bar{r})=-\frac{\bar{r}\left[\bar{r}-\bar{\gamma}\left(\beta_{11}-\beta_{12}\right)\right]\left(\beta_{12}+\beta_{21}\right)}{\left(\beta_{11}-\beta_{12}\right)^{2}}<0$

It follows that one characteristic root is negative and two characteristic roots are positive, with one equal to $\bar{\delta}$. The balanced growth path is saddle-point stable regardless of the factor intensity difference. This result corresponds to that obtained by d'Autume and Michel [1] and Bond et al. [10].

When external effects are considered, this saddle-point property may no longer hold and local indeterminacy becomes a possible outcome. Moreover, if multiplicity holds, we need to compare the properties of two stationary BGPs. The following fundamental Lemma provides some links between the product of characteristic roots $\mathcal{D}(r)$ and the slopes of $\mathcal{F}(r)$ and $\mathcal{G}(r)$ at the stationary BGP. This result is the central argument of our stability analysis.

Lemma 3. Under Assumptions 1-2, consider $r^{*}$ and $\rho^{*}$ as defined by equations (29). Then if $\bar{r}=r^{*}, \mathcal{D}(\bar{r})=0$ and for any $\bar{r} \neq r^{*}, \mathcal{D}(\bar{r})<(>) 0$ if and only if $\mathcal{F}^{\prime}(\bar{r})-\mathcal{G}^{\prime}(\bar{r})>(<) 0$.

Proof. See Appendix 6.7. 
This lemma also allows to provide a set of necessary conditions for the occurrence of local indeterminacy of order 3 for a given BGP $(\bar{r}, \bar{\gamma})$. Indeed, the existence of three characteristic roots with a negative real part requires $\mathcal{T}(\bar{r})<0, \mathcal{S}(\bar{r})>0$ and $\mathcal{D}(\bar{r})<0$. As Assumptions 1-2 imply $\left[\bar{r}-\gamma\left(\beta_{11}-\right.\right.$ $\left.\left.\beta_{12}\right)\right]>0$, we easily derive from Proposition 2 the following result:

Lemma 4. Under Assumptions 1-2, consider a stationary BGP as given by $(\bar{r}, \bar{\gamma})$. Necessary conditions for the existence of local indeterminacy of order 3 are: $b_{1}-b_{2}<0, \mathcal{F}^{\prime}(\bar{r})-\mathcal{G}^{\prime}(\bar{r})>0$ and $(1-\sigma) /\left(\beta_{11}-\beta_{12}\right)>0$.

The third condition implies that the existence of local indeterminacy of order 3 can only be obtained in two particular configurations concerning the elasticity of intertemporal substitution in consumption and the physical capital intensity difference across the two sectors, namely:

i) when the elasticity of intertemporal substitution in consumption is lower than one, i.e. $1 / \sigma<1$, and the human capital sector is more intensive in physical capital than the final good sector, i.e. $\beta_{11}<\beta_{12}$, or

ii) when the elasticity of intertemporal substitution in consumption is larger than one, i.e. $1 / \sigma>1$, and the final good sector is more intensive in physical capital than the human capital sector, i.e. $\beta_{11}>\beta_{12}$.

Let us provide now the local stability analysis of the BGP. We start by a simple benchmark formulation with a logarythmic utility function in which only a local indeterminacy of order 2 can occur.

\subsection{A benchmark formulation with unitary intertemporal elasticity of substitution}

If the intantaneous utility function $u(c)$ is logarythmic, i.e. $\sigma=1$, the intertemporal elasticity of substitution is unitary and we derive from Proposition 2 that $\mathcal{Y}(\bar{r})=0$ and $\mathcal{S}(\bar{r})=\bar{\delta}(\tilde{\mathcal{T}}(\bar{r})+\bar{\delta})+\tilde{\mathcal{D}}(\bar{r})$. The characteristic polynomial can be simplified as

$$
g(\lambda)=(\lambda-\bar{\delta})\left[\lambda^{2}-\lambda(\tilde{\mathcal{T}}(\bar{r})+\bar{\delta})+\tilde{\mathcal{D}}(\bar{r})\right]=0
$$

and one root is thus equal to $\bar{\delta}>0$ while the sign of the two other roots depends on the signs of $\tilde{\mathcal{D}}(\bar{r})$ and $\tilde{\mathcal{T}}(\bar{r})+\bar{\delta}$. Local indeterminacy is obtained 
if $\tilde{\mathcal{D}}(\bar{r})>0$ and $\tilde{\mathcal{T}}(\bar{r})+\bar{\delta}<0,{ }^{15}$ while saddle-point stability follows from $\tilde{\mathcal{D}}(\bar{r})<0$. The sign of $\tilde{\mathcal{D}}(\bar{r})$ can be easily analysed from Lemma 3 . The sign of $\tilde{\mathcal{T}}(\bar{r})+\bar{\delta}$ depends on the Cobb-Douglas technological exponents. It follows indeed that $\tilde{\mathcal{T}}(\bar{r})+\bar{\delta}$ will be positive if $b_{2}-b_{1}<0$ and can be negative if $b_{2}-b_{1}>0$ and the capital intensity difference at the private level $\beta_{11}-\beta_{12}$ is close enough to zero. A necessary condition for local indeterminacy is then $b_{2}-b_{1}>0$.

Note from (30) that the bound $\rho_{1}$ cannot be defined so that $\rho_{2}^{*}=\rho_{2}$. Moreover, when $\rho^{*}$, as defined by equation (29), satisfies $r^{*}-\rho^{*}>0$, we necessarily have $\rho_{2}<\rho^{*}$.

Theorem 3. Under Assumptions 1-2, let $\sigma=1$ and $\delta=r-\gamma$. When $\mu \leq$ 1 , any steady state is locally determinate regardless of the factor intensity difference. On the contrary, when $\mu>1$ the following cases hold:

1 - Let $\beta_{11}<\beta_{12}$, i.e. the final good is intensive in human capital at the private level. Then:

i) If $\mu \in\left(1, \beta_{12} / \beta_{11}\right)$, the steady states $\left(\bar{r}_{1}, \bar{\gamma}_{1}\right)$ and $\left(\bar{r}_{2}, \bar{\gamma}_{2}\right)$ are such that $\bar{\gamma}_{2}>\bar{\gamma}_{1}$ and $\left(\bar{r}_{1}, \bar{\gamma}_{1}\right)$ is locally indeterminate of order 2 if and only if $b_{2}>b_{1}, \tilde{\mathcal{T}}\left(\bar{r}_{1}\right)+\bar{\delta}_{1}<0$ and $\rho \in\left(\rho_{2}, \rho^{*}\right),{ }^{16}$ while $\left(\bar{r}_{2}, \bar{\gamma}_{2}\right)$ is saddle-point stable for any $\rho<\rho^{*}$.

ii) If $\mu \geq \beta_{12} / \beta_{11}$ and $\rho<\rho_{2}$, the unique steady state $(\bar{r}, \bar{\gamma})$ is saddlepoint stable.

2 - If $\beta_{11}>\beta_{12}$, i.e. the final good is intensive in physical capital at the private level, and $\rho<\rho_{2}$, then the unique steady state $(\bar{r}, \bar{\gamma})$ is saddle-point stable.

Proof. See Appendix 6.8.

This Theorem, and its illustration on the bifurcation diagram in Figure 3 , shows that with logarythmic preferences, local indeterminacy can only appear in presence of multiple stationary BGPs when the final good is intensive in human capital at the private level. This condition is the same

\footnotetext{
${ }^{15} \mathrm{~A}$ positive value for $\tilde{\mathcal{T}}(\bar{r})+\bar{\delta}$ implies local instability of the steady state. Note also that crossing the frontier $\tilde{\mathcal{T}}(\bar{r})+\bar{\delta}=0$ with $\tilde{\mathcal{D}}(\bar{r})>0$ implies the generic existence of a Hopf bifurcation and periodic cycles.

${ }^{16}\left(\bar{r}_{1}, \bar{\gamma}_{1}\right)$ no longer exists as soon as $\rho \leq \rho_{2}$.
} 

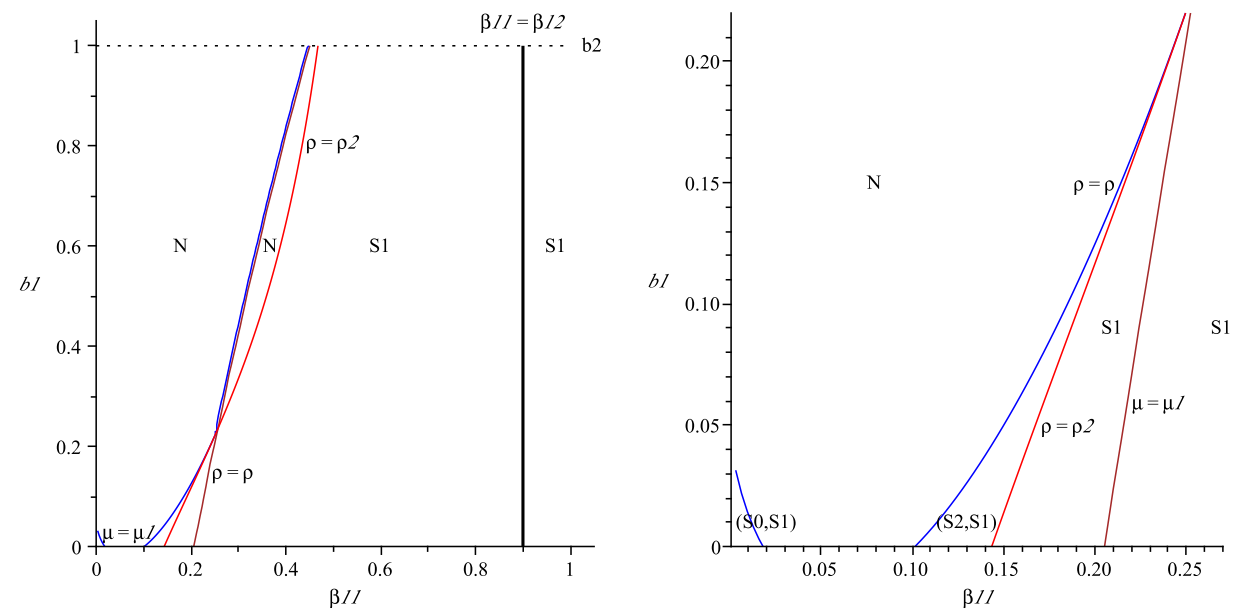

Figure 3: Bifurcation diagram for $\sigma=1, \beta_{22}=0.1, b_{2}=1$, and $\rho=0.39072$. The left panel shows curves $\beta_{11}=\beta_{12}, b_{1}=b_{2}, \mu=\mu_{1}>1, \rho=\rho^{*}$ and $\rho=\rho_{2}$ which separate several stability regions: in $N$ there is no BGP, in $S_{1}$ there is a unique saddle point stable BGP, and in regions $\left(S_{1}, S_{0}\right)$ and $\left(S_{1}, S_{2}\right)$ there are two BGP's, where $\bar{\gamma}_{1}$ is saddle-point stable and $\bar{\gamma}_{2}$ is unstable $\left(S_{0}\right)$ or locally indeterminate of order $2\left(S_{2}\right)$. The right panel zooms the bottom left corner of the left panel.

as the one obtained by Bond et al. [10] in a similar two-sector model with taxes and by Benhabib et al. [6], Mino [21] or Nishimura and Venditti [25] in a similar two-sector model with sector specific externalities. Note however that contrary to these papers, we give conditions for the existence of multiple stationary BGPs and we show with Theorem 3 that local and global indeterminacy are closely related since a two-dimensional stable manifold only appears in the presence of two stationary balanced growth rates. It is worth noting then that with a unitary elasticity of intertemporal substitution in consumption, economies with two stationary BGPs can be characterized by a poverty trap. Indeed, when stable, the low growth BGP $\bar{\gamma}_{1}$ is locally indeterminate while the high growth BGP $\bar{\gamma}_{2}$ is saddle-point stable.

In the next section we extend these results to the general case of a non unitary intertemporal elasticity of substitution for consumption.

\subsection{A general formulation}

In the general case $\sigma>0$ with $\sigma \neq 1$, we will prove that local and global indeterminacy can appear for any capital intensity configuration, i.e. if the 
final good is intensive in physical capital at the private level, or if the final good is intensive in human capital at the private level.

Contrary to the previous section, the analysis is more difficult since the characteristic polynomial of degree 3 does not have a trivial root. The local dynamic properties of the stationary BGP will of course depend on the sign of $\mathcal{D}(\bar{r}), \mathcal{S}(\bar{r})$ and $\mathcal{T}(\bar{r}) . \mathcal{D}(\bar{r})$ can be easily analysed from Lemma 3 . The sign of $\mathcal{T}(\bar{r})$ depends on the Cobb-Douglas exponents: $\mathcal{T}(\bar{r})$ will be positive if $b_{2}-b_{1}<0$ and can be negative if $b_{2}-b_{1}>0$ and the capital intensity difference at the private level $\beta_{11}-\beta_{12}$ is close enough to zero. The sign of the difference $\mathcal{D}(\bar{r})-\mathcal{S}(\bar{r}) \mathcal{T}(\bar{r})$ will also appear to be very important but remains difficult to study. Note however that from Proposition 2 we have

$$
\mathcal{D}(\bar{r})-\mathcal{S}(\bar{r}) \mathcal{T}(\bar{r})=-(\bar{\delta}+\tilde{\mathcal{T}}(\bar{r})) \tilde{\mathcal{D}}(\bar{r})-\mathcal{T}(\bar{r})[\bar{\delta}(\bar{\delta}+\tilde{\mathcal{T}}(\bar{r}))+\mathcal{Y}(\bar{r})]
$$

It follows that when $\tilde{\mathcal{D}}(\bar{r}), \mathcal{Y}(\bar{r})$ and $\tilde{\mathcal{T}}(\bar{r})$ are positive, the difference $\mathcal{D}(\bar{r})-$ $\mathcal{S}(\bar{r}) \mathcal{T}(\bar{r})$ is negative.

Note finally that Definition 1, which provides a distinction between local indeterminacy of order 2 and local indeterminacy of order 3, allows to simplify the exposition of the results. Indeed, it will be easy to see in the following Theorems that as soon as the conditions for local indeterminacy of order 2 do not hold, the stationary BGP is locally unstable. Similarly, as soon as the conditions for local indeterminacy of order 3 do not hold, the stationary BGP is saddle-point stable.

We start by the configuration in which the final good is intensive in human capital at the private level.

Theorem 4. Under Assumptions 1-2, let $\beta_{11}<\beta_{12}$, and consider the bounds $\mu_{1}, \mu_{2}$ as defined by (28). Then the following cases hold:

1 - Assume first that $\sigma>1$.

i) If $\mu \leq 1$ and $\rho<\rho_{2}^{*}$, the steady state $(\bar{r}, \bar{\gamma})$ is locally unstable.

ii) Let $\mu \in\left(1, \mu_{1}\right)$.

a) When $\rho \in\left(\rho_{2}^{*}, \rho^{*}\right)$, the steady states $\left(\bar{r}_{1}, \bar{\gamma}_{1}\right)$ and $\left(\bar{r}_{2}, \bar{\gamma}_{2}\right)$ are such that $\bar{\gamma}_{2}>\bar{\gamma}_{1}$ and $\left(\bar{r}_{1}, \bar{\gamma}_{1}\right)$ is locally indeterminate of order 2 if $b_{2}>b_{1}$ and $\mathcal{T}\left(\bar{r}_{1}\right)<0$, or $\mathcal{T}\left(\bar{r}_{1}\right)>0$ and $\mathcal{D}\left(\bar{r}_{1}\right)-\mathcal{S}\left(\bar{r}_{1}\right) \mathcal{T}\left(\bar{r}_{1}\right)>0$, while $\left(\bar{r}_{2}, \bar{\gamma}_{2}\right)$ is locally indeterminate of order 3 if and only if $b_{2}>b_{1}, \mathcal{T}\left(\bar{r}_{2}\right)<0$ and 
$\mathcal{D}\left(\bar{r}_{2}\right)-\mathcal{S}\left(\bar{r}_{2}\right) \mathcal{T}\left(\bar{r}_{2}\right)>0$.

b) When $\rho \leq \rho_{2}^{*},(\bar{r}, \bar{\gamma})$ is locally indeterminate of order 3 if and only if $b_{2}>b_{1}, \mathcal{T}(\bar{r})<0$ and $\mathcal{D}(\bar{r})-\mathcal{S}(\bar{r}) \mathcal{T}(\bar{r})>0$.

iii) Let $\mu \geq \mu_{1}$ and $\rho<\rho_{2}^{*}$. $(\bar{r}, \bar{\gamma})$ is locally indeterminate of order 3 if and only if $b_{2}>b_{1}, \mathcal{T}(\bar{r})<0$ and $\mathcal{D}(\bar{r})-\mathcal{S}(\bar{r}) \mathcal{T}(\bar{r})>0$.

2 - Assume now that $\sigma<1$.

i) Let $\mu \leq 1$ and $\rho \in\left(\rho_{1}^{*}, \rho_{2}^{*}\right)$. $(\bar{r}, \bar{\gamma})$ is locally indeterminate of order 2 if and only if $\mathcal{D}(\bar{r})-\mathcal{S}(\bar{r}) \mathcal{T}(\bar{r})>0$.

ii) Let $\mu \in\left(1, \mu_{2}\right)$ and $\rho \in\left(\rho_{1}^{*}, \rho_{2}^{*}\right) .(\bar{r}, \bar{\gamma})$ is locally indeterminate of order 2 if $b_{2}>b_{1}$ and $\mathcal{T}(\bar{r})<0$, or $\mathcal{T}(\bar{r})>0$ and $\mathcal{D}(\bar{r})-\mathcal{S}(\bar{r}) \mathcal{T}(\bar{r})>0$.

iii) Let $\mu \in\left(\mu_{2}, \mu_{1}\right)$.

a) When $\rho \in\left(\rho_{2}^{*}, \rho^{*}\right)$, the steady states $\left(\bar{r}_{1}, \bar{\gamma}_{1}\right)$ and $\left(\bar{r}_{2}, \bar{\gamma}_{2}\right)$ are such that $\bar{\gamma}_{2}>\bar{\gamma}_{1}$ and $\left(\bar{r}_{1}, \bar{\gamma}_{1}\right)$ is locally indeterminate of order 2 if $b_{2}>b_{1}$ and $\mathcal{T}\left(\bar{r}_{1}\right)<0$, or $\mathcal{T}\left(\bar{r}_{1}\right)>0$ and $\mathcal{D}\left(\bar{r}_{1}\right)-\mathcal{S}\left(\bar{r}_{1}\right) \mathcal{T}\left(\bar{r}_{1}\right)>0$, while $\left(\bar{r}_{2}, \bar{\gamma}_{2}\right)$ is saddle-point stable.

b) When $\rho \in\left(\rho_{1}^{*}, \rho_{2}^{*}\right]$ and $\sigma \in\left(1-\left(\beta_{12} / \beta_{11}\right)^{1 /(1-\mu)}, 1\right),(\bar{r}, \bar{\gamma})$ is saddlepoint stable.

c) When $\rho \in\left(\rho_{1}^{*}, \rho_{2}^{*}\right]$ and $\sigma \in\left(0,1-\left(\beta_{12} / \beta_{11}\right)^{1 /(1-\mu)}\right),(\bar{r}, \bar{\gamma})$ is locally indeterminate of order 2 if $b_{2}>b_{1}$ and $\mathcal{T}(\bar{r})<0$, or $\mathcal{T}(\bar{r})>0$ and $\mathcal{D}(\bar{r})-\mathcal{S}(\bar{r}) \mathcal{T}(\bar{r})>0$.

iv) If $\mu \geq \mu_{1}$ and $\rho \in\left(\rho_{1}^{*}, \rho_{2}^{*}\right),(\bar{r}, \bar{\gamma})$ is saddle-point stable.

Proof. See Appendix 6.9.

Theorem 4 shows that the local stability properties are sensitive with respect to the elasticity of intertemporal substitution in consumption. When the elasticity is less than unity, i.e. $1 / \sigma<1$, external effects such that $\mu \leq 1$ imply local instability. Following Santos [29] this means that there may not exist any equilibrium path. ${ }^{17}$ On the contrary, when externalities are such that $\mu>1$, local and global indeterminacy can occur. An interesting new result is provided by case $1-i i) a$ ) in which two distinct locally indeterminate stationary BGPs can co-exist. Such a globally indeterminate configuration has never been illustrated previously. Moreover, and contrary to the config-

\footnotetext{
${ }^{17}$ There is a BGP only if $\left(k_{1}(0), k_{2}(0)\right)$ belong to a particular one-dimensional manifold.
} 
uration 1-i) in Theorem 3, the high growth BGP $\bar{\gamma}_{2}$ can be more attractive than the low growth BGP $\bar{\gamma}_{1}$ as it can be locally indeterminate of order 3 while the other is locally indeterminate of order 2 . In this case, the poverty trap can be avoided by choosing an adequate equilibrium path. Note however that the high growth BGP $\bar{\gamma}_{2}$ can also be saddle-point stable while the low growth BGP $\bar{\gamma}_{1}$ is locally indeterminate of order 2 . In such a case the poverty trap cannot be avoided. These configurations are illustrated in the following figure:

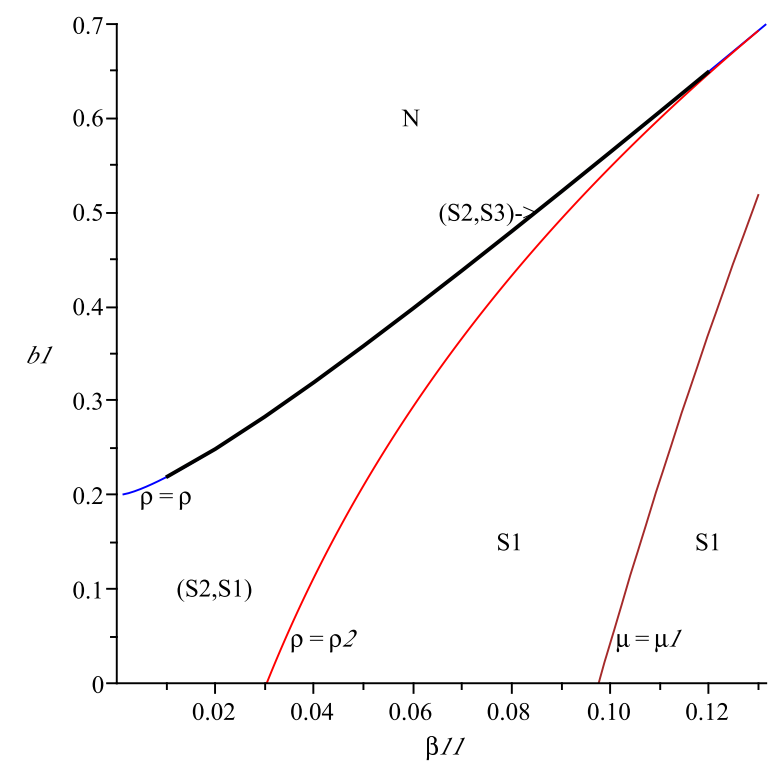

Figure 4: Bifurcation diagram for $\sigma=1.15>1, \beta_{22}=0.66, b_{2}=1$, and $\rho=0.165$. The labels for the curves and regions are as in figure $3 .\left(S_{2}, S_{3}\right)$ is a new case where $\bar{\gamma}_{1}$ is indeterminate of order 2 and $\bar{\gamma}_{2}$ is indeterminate of order 3 .

When the elasticity of intertemporal substitution is on the contrary greater than unity, i.e. $1 / \sigma>1$, local and global indeterminacy are compatible with both $\mu \leq 1$ and $\mu>1$. However, when global indeterminay arises, one stationary balanced growth path is necessarily saddle-point stable. Moreover, global indeterminacy is now intimately related to the existence of a poverty trap as in the case of Theorem 3 . When stable, the low growth BGP $\bar{\gamma}_{1}$ is locally indeterminate while the high growth BGP $\bar{\gamma}_{2}$ is saddle-point stable. 
We consider finally the configuration in which the final good is intensive in physical capital at the private level.

Theorem 5. Under Assumptions 1-2, let $\beta_{11}>\beta_{12}$, and consider the bounds $\mu_{1}, \mu_{2}$ as defined by (28). Then the following cases hold:

1 - Assume first that $\sigma>1$.

i) Let $\mu \leq \mu_{1}$ and $\rho<\rho_{2}^{*}$. $(\bar{r}, \bar{\gamma})$ is locally indeterminate of order 2 if and only if $\mathcal{D}(\bar{r})-\mathcal{S}(\bar{r}) \mathcal{T}(\bar{r})>0$.

ii) Let $\mu \in\left(\mu_{1}, 1\right)$.

a) When $\rho \in\left(\rho_{2}^{*}, \rho^{*}\right)$, the steady states $\left(\bar{r}_{1}, \bar{\gamma}_{1}\right)$ and $\left(\bar{r}_{2}, \bar{\gamma}_{2}\right)$ are such that $\bar{\gamma}_{2}>\bar{\gamma}_{1}$ and $\left(\bar{r}_{1}, \bar{\gamma}_{1}\right)$ is saddle-point stable, while $\left(\bar{r}_{2}, \bar{\gamma}_{2}\right)$ is locally indeterminate of order 2 if and only if $\mathcal{D}\left(\bar{r}_{2}\right)-\mathcal{S}\left(\bar{r}_{2}\right) \mathcal{T}\left(\bar{r}_{2}\right)>0$.

b) When $\rho \leq \rho_{2}^{*},(\bar{r}, \bar{\gamma})$ is locally indeterminate of order 2 if and only if $\mathcal{D}(\bar{r})-\mathcal{S}(\bar{r}) \mathcal{T}(\bar{r})>0$.

iii) If $\mu \geq 1$ and $\rho<\rho_{2}^{*},(\bar{r}, \bar{\gamma})$ is saddle-point stable.

2 - Assume now that $\sigma<1$.

i) If $\mu<1$, any steady state is locally determinate.

ii) Let $\mu \geq 1$ and $\rho \in\left(\rho_{1}^{*}, \rho_{2}^{*}\right)$. $(\bar{r}, \bar{\gamma})$ is locally indeterminate of order 3 if and only if $b_{2}>b_{1}, \mathcal{T}(\bar{r})<0$ and $\mathcal{D}(\bar{r})-\mathcal{S}(\bar{r}) \mathcal{T}(\bar{r})>0$.

Proof. See Appendix 6.10.

When compared with Theorem 4, Theorem 5 shows that there exists a complementarity between the previous case with a final good intensive in human capital at the private level and the current case with a final good intensive in physical capital at the private level. When the elasticity of intertemporal substitution in consumption is less than unity, the existence of sunspot equilibria requires external effects with $\mu<1$ while if $\mu \geq 1$, the steady state is always saddle-point. On the contrary, when the elasticity of substitution is greater than unity, local indeterminacy is compatible with external effects such that $\mu \geq 1$. Note however that in this case, uniqueness of the stationary balanced growth rate is always satisfied.

Moreover, contrary to Theorem 4 , when $1 / \sigma<1$, global indeterminacy is always associated with a high growth BGP $\bar{\gamma}_{2}$ which is more attractive than the low growth BGP $\bar{\gamma}_{1}$ as it is locally indeterminate of order 2 while the 
other is saddle-point stable. Here the poverty trap can always be avoided by choosing an adequate equilibrium path. Considering Theorems 3, 4 and 5 in perspective, we then derive the following conclusion:

Corollary 2. Under Assumptions 1-2, global indeterminacy is always associated with the existence of a poverty trap when $1 / \sigma \geq 1$, i.e. there exists a set of initial conditions of positive measure from which the equilibrium path converges to the low growth BGP. On the contrary, when $1 / \sigma<1$, global indeterminacy can be characterized by a high growth BGP which is more attractive than the low growth BGP, i.e. there exists a set of initial conditions of positive measure from which the equilibrium path converges to the high growth BGP. This is always the case when the final good is intensive in physical capital at the private level, i.e. $\beta_{11}>\beta_{12}$.

This corollary shows that a poverty trap always exists when the elasticity of intertemporal substitution in consumption is larger than unity, i.e. $1 / \sigma \geq 1$. Indeed, the high growth BGP is saddle-point stable while the low growth BGP is locally indeterminate of order 2. This conclusion is drastically different from the one obtained by García-Belenguer [15] who shows that poverty traps occur when the elasticity of intertemporal substitution is lower than unity.

Corollary 2 shows however that when $1 / \sigma<1$, the poverty trap can be avoided as the high growth BGP is either locally indeterminate of order 3 or saddle-point stable while the low growth BGP is respectively either locally indeterminate of order 2 or totally unstable. In this case, the dimension of the set of initial conditions from which the equilibrium path converges towards the high growth BGP can be larger than these of the set of initial conditions from which the equilibrium path converges to the low growth BGP. In other words, for given initial levels of physical and human capital, there can exist a set of initial prices giving rise to equilibrium paths converging to the high growth BGP whose positive measure is larger than these of the set of initial prices giving rise to equilibrium paths converging to the low growth BGP. However a poverty trap may still exist when $\beta_{11}<\beta_{12}$ if the high growth BGP is saddle-point stable. Figure 4 precisely illustrates a case 
with in which $\beta_{11}<\beta_{12}$ and the high growth BGP is either indeterminate of order 3 or saddle point stable and the low growth BGP is indeterminate of order 2 . Note that the last configuration is much more robust.

\section{Concluding comments}

We have studied the dynamics of a two-sector endogenous growth model with physical and human capital accumulation and economy-wide external effects in the production function of both sectors. Local and global indeterminacy have been discussed through the existence of multiple stationary balanced growth paths and through the local stability analysis of each long run position.

We have shown that two stationary balanced growth paths easily arise for a large set of parameters configurations which includes both signs for the capital intensity difference at the private level and values of the elasticity of intertemporal substitution in consumption lower or larger than unity. We have also proved that for a given stationary solution, two kinds of local indeterminacy can occur: a local indeterminacy of order 2 in which the stable manifold has dimension 2, and a local indeterminacy of order 3 in which the stable manifold has dimension 3 . This last case is particularly interesting as we prove that in a configuration with two stationary solutions, one can be locally indeterminate of order 2 while the other is locally indeterminate of order 3. This type of generalized local and global indeterminacy has never been illustrated in the literature. Moreover, we have shown that local indeterminacy can be obtained independently of the sign of the capital intensity difference at the private level and the value of the elasticity of intertemporal substitution in consumption. From this point of view, we show that in our model, local and global indeterminacy are more robust properties than in most of the models considered in the literature.

Finally, we have discussed our main conclusions in terms of their implications for development economics. We have proved that global indeterminacy is always associated with the existence of a poverty trap when the elasticity of intertemporal substitution in consumption is larger than unity. 


\section{References}

[1] d'Autume, A. and P. Michel (1992): "The New Growth Theories. Part 1: Persistence, Hysteresis and Multiple Equilibria," DP 92.20 (in french), University of Paris 1.

[2] Azariadis, C. and J. Stachurski (2005): "Poverty Traps," in Handbook of Economic Growth, Vol.1, P. Aghion and S. Durlauf (eds.), NorthHolland, Amsterdam,295-384.

[3] Barinci, J.P. and J.P. Drugeon (1999): "Assessing Local Indeterminacy in Three-Dimensional Dynamical Systems: a Geometric Perspective," mimeo, EUREQua.

[4] Basu, S., and J. Fernald (1997): "Returns to Scale in US Production: Estimates and Implications," Journal of Political Economy, 105, 249283.

[5] Ben-Gad, M. (2003): "Fiscal Policy and Indeterminacy in Models of Endogenous Growth," Journal of Economic Theory, 108, 322-344.

[6] Benhabib, J., Meng, Q. and K. Nishimura (2000): "Indeterminacy under Constant Returns in Multisector Economies," Econometrica, 68, 1541-1548.

[7] Benhabib, J. and R. Perli (1994): "Uniqueness and Indeterminacy: Transitional Dynamics in a Model of Endogenous Growth," Journal of Economic Theory, 63, 113-142.

[8] Bloise, G. (2001): "A Geometric Approach to Sunspot Equilibria," Journal of Economic Theory, 101, 519-539.

[9] Bloise, G. and F. Magris (2000): "Sunspot Equilibria in a Monetary Economy with Capital Accumulation," CORE DP 0040.

[10] Bond, E., Wang, P. and C. Yip (1996): "A General Two-Sector Model of Endogenous Growth with Human and Physical Capital: Balanced 
Growth and Transitional Dynamics," Journal of Economic Theory, 68, 149-173.

[11] Caballe, J. and M. Santos (1993): "On Endogenous Growth with Physical and Human Capital," Journal of Political Economy, 101, 1042-1068.

[12] Chamley, C. (1993): "Externalities and Dynamics in Models of Learning-by-Doing," International Economic Review, 34, 583-609.

[13] Drugeon, J.P. (2008): "On Intersectoral Asymmetries in Factors Substitutability, "Equilibrium Production Possibility Frontiers" and the Emergence of Indeterminacies," Journal of Mathematical Economics $44,277-315$

[14] Drugeon, J.P., Poulsen, O. and A. Venditti (2003): "On Intersectoral Allocations, Factors Substitutability and Multiple Long-Run Growth Paths," Economic Theory, 21, 175-183.

[15] García-Belenguer, F. (2007): "Stability, Global Dynamics and Markov Equilibrium in Models of Endogenous Economic Growth," Journal of Economic Theory, 136, 392-416.

[16] Ladrón-de-Guevara, A., Ortigueira, S. and M. Santos (1997): "Equilibrium Dynamics in Two Sector Models of Endogenous Growth," Journal of Economic Dynamics and Control, 21, 115-143.

[17] Ladrón-de-Guevara, A., Ortigueira, S. and M. Santos (1999): "A Two Sector Model of Endogenous Growth with Leisure," Review of Economic Studies, 66, 609-631.

[18] Leonard, D. and N. Van Long (1992): Optimal Control Theory and Static Maximization in Economics, Cambridge University Press.

[19] Lucas, R. (1988): "On the Mechanics of Economic Development," Journal of Monetary Economics, 22, 3-42.

[20] Mattana, P., Nishimura., K. and T. Shigoka (2009): "Homoclinic Bifurcation and Global Indeterminacy of Equilibrium in a Two-Sector 
Endogenous Growth Model," International Journal of Economic Theory, 5, 25-47.

[21] Mino, K. (2001): "Indeterminacy and Endogenous Growth with Social Constant Returns," Journal of Economic Theory, 97, 203-222.

[22] Mitra, T. (1998): "On Equilibrium Dynamics under Externalities in a Model of Economic Development," Japanese Economic Review, 49, 85-107.

[23] Mulligan, C. and X. Sala-i-Martin (1993): "Transitional Dynamics in Two Sector Models of Endogenous Growth," Quarterly Journal of Economics, 103, 739-773.

[24] Nishimura, K., Takahashi, H. and A. Venditti (2009): "Global Externalities, Endogenous Growth and Sunspot Fluctuations," Advanced Studies in Pure Mathematics, 53, 227-238.

[25] Nishimura, K., and A. Venditti (2004): "Indeterminacy and the Role of Factor Substitutability," Macroeconomic Dynamics, 8, 436-465.

[26] Raurich, X. (2001): "Indeterminacy and Government Spending in a Two-Sector Model of Endogenous Growth," Review of Economic Dynamics, 4, 210-229.

[27] Rebelo, S. (1991): "Long-Run Policy Analysis and Long-Run Growth," Journal of Political Economy, 99, 500-521.

[28] Romer, P. (1986): "Increasing Returns and Long-Run Growth," Journal of Political Economy, 94, 1002-1037.

[29] Santos, M. (2002): "On Non-Existence of Markov Equilibria in Competitive-Market Economies," Journal of Economic Theory, 105, 73-98.

[30] Woodford, M. (1986): "Stationary Sunspot Equilibria: the Case of Small Fluctuations Around a Deterministic Steady Sate," mimeo, University of Chicago. 
[31] Xie, D. (1994): "Divergence in Economic Performance: Transitional Dynamics with Multiple Equilibria," Journal of Economic Theory, 63, 97-112.

\section{Appendix}

\subsection{Proof of Lemma 1}

From (4), we have

$$
K_{i j} / Y_{j} \equiv a_{i j}=P_{j} \beta_{i j} / R_{i}
$$

We call $a_{i j}$ the input coefficient from the private viewpoint. Denoting $A=$ $\left[a_{i j}\right], Y=\left(Y_{1}, Y_{2}\right)^{\prime}$ and $K=\left(K_{1}, K_{2}\right)^{\prime}$, it follows from (4)-(5) that $A Y=K$. Moreover, denoting

$$
P=\left(\begin{array}{cc}
P_{1} & 0 \\
0 & P_{2}
\end{array}\right) \text { and } R=\left(\begin{array}{cc}
R_{1} & 0 \\
0 & R_{2}
\end{array}\right)
$$

we have $A=R^{-1} B P$. It follows from the constant returns to scale property of the individual technologies that the final good is intensive in physical (human) capital at the private level, i.e. $a_{11} / a_{21}>(<) a_{12} / a_{22}$, if and only if $\beta_{11}-\beta_{12}>(<) 0$. Moreover under Assumption 1, $A$ is invertible and

$$
Y=A^{-1} K \equiv\left(\begin{array}{cc}
\alpha_{11} & \alpha_{12} \\
\alpha_{21} & \alpha_{22}
\end{array}\right) K=\left(\begin{array}{cc}
R_{1} \frac{\psi_{11}}{P_{1}} & R_{2} \frac{\psi_{12}}{P_{1}} \\
R_{1} \frac{\psi_{21}}{P_{2}} & R_{2} \frac{\psi_{22}}{P_{2}}
\end{array}\right)\left(\begin{array}{c}
K_{1} \\
K_{2}
\end{array}\right)
$$

From this we get the optimal private allocations

$$
K_{i j}=\frac{\beta_{i j}}{R_{i}}\left(\psi_{j 1} R_{1} K_{1}+\psi_{j 2} R_{2} K_{2}\right), \text { for } i, j=1,2
$$

which are linear functions of the total private amounts of the two capital stocks. The result follows from using again (4) and the fact that the private returns to scale are constant.

\subsection{Proof of Lemma 2}

Let $k=k_{1} / k_{2}=K_{1} / K_{2}$. Proposition 1 and equation (12) give

$$
\pi=\left[\left(\beta_{1}^{*} / \beta_{2}^{*}\right) k^{b_{1}-b_{2}}\right]^{1 /\left(\beta_{12}+\beta_{21}\right)}
$$

Substituting this into equation (10) gives 


$$
r=\left[\left(\beta_{1}^{*}\right)^{\beta_{12}}\left(\beta_{2}^{*}\right)^{\beta_{21}} k^{\beta_{12} b_{1}+\beta_{21} b_{2}}\right]^{\frac{1}{\beta_{12}+\beta_{21}}}
$$

Moreover, after solving equations (13) and (14) we get:

$$
\sigma \beta_{21} r=\frac{\pi}{k}\left[\left(\beta_{11}(\sigma-1)+\beta_{12}\right) r+\rho\left(\beta_{11}-\beta_{12}\right)\right]
$$

Considering (24) and (25), we derive

$$
\frac{\pi}{k}=\left[\left(\beta_{1}^{*}\right)^{\beta_{12}+b_{2}}\left(\beta_{2}^{*}\right)^{\beta_{21}-b_{1}} r^{-\left(\beta_{12}+\beta_{21}+b_{2}-b_{1}\right)}\right]^{1 /\left(\beta_{12} b_{1}+\beta_{21} b_{2}\right)}
$$

Substituting this into (26) finally gives the result.

\subsection{Proof of Theorem 1}

Note first that $\mathcal{F}(r)$ is monotone increasing (decreasing) if and only if $\mu>0$ $(\mu<0)$ and concave (convex) if and only if $\mu \in(0,1)(\mu \in(-\infty, 0) \cup$ $(1,+\infty))$. Moreover

$$
\begin{aligned}
\mu>0 & \Leftrightarrow \beta_{12}\left(1+b_{1}\right)+\beta_{21}\left(1+b_{2}\right)>b_{1}-b_{2} \\
\mu>1 & \Leftrightarrow \beta_{12}+\beta_{21}>b_{1}-b_{2}
\end{aligned}
$$

When the final good is intensive in human capital at the private level, i.e. $\beta_{11}<\beta_{12}, \mathcal{G}(r)$ is monotone increasing for any value of $\sigma>0$. On the contrary, denoting $\hat{\sigma}=\left(\beta_{11}-\beta_{12}\right) / \beta_{11}$, when the final good is intensive in physical capital at the private level, i.e. $\beta_{11}>\beta_{12}, \mathcal{G}(r)$ is monotone increasing (decreasing) if and only if $\sigma>\hat{\sigma}(\sigma \in(0, \hat{\sigma}))$. Our strategy for studying the existence and multiplicity of stationary BGPs is based on the properties of these two functions. Indeed if $\mathcal{F}(r)$ and $\mathcal{G}(r)$ have opposite slopes, there exists at most one stationary balanced growth rate. Some boundary conditions can then guarantee existence. On the contrary, if both $\mathcal{F}(r)$ and $\mathcal{G}(r)$ have positive or negative slopes, we can find a tangency point between these two functions by solving simultaneously $\mathcal{F}(r)=\mathcal{G}(r)$ and $\mathcal{F}^{\prime}(r)=\mathcal{G}^{\prime}(r)$ with respect to $r$ and $\rho$. This gives the following values:

$$
r^{*}=\left(\frac{\beta_{11}(\sigma-1)+\beta_{12}}{\mu \sigma \beta_{21} \tilde{\beta}}\right)^{\frac{1}{\mu-1}}, \quad \rho^{*}=\frac{(1-\mu)\left[\beta_{11}(\sigma-1)+\beta_{12}\right]}{\mu\left(\beta_{11}-\beta_{12}\right)} r^{*}
$$

When $\rho=\rho^{*}, r^{*}$ is a tangency point between $\mathcal{F}(r)$ and $\mathcal{G}(r)$. Additional conditions on the parameters of the model that will be necessary to get admissible values for $r^{*}$ and $\rho^{*}$ are straightforward from (29), namely:

i) $\mu>1$ and $\beta_{12}>\beta_{11}$, or 
ii) $\mu \in(0,1)$ and $\beta_{12} \in\left(\beta_{11}(\sigma-1), \beta_{11}\right)$, or

iii) $\mu<0$ and $\beta_{12}<\beta_{11}(\sigma-1)$.

When such an admissible tangency point exists, we define the corresponding growth rate as $\gamma^{*}=\left(r^{*}-\rho^{*}\right) / \sigma$. Under Assumption 2, as $\gamma=(r-\rho) / \sigma$, the long run real rate of return should verify the condition $\rho-(1-\sigma) r>0$. Moreover, since $\gamma>0$ we need to have $r>\rho$. It follows that $r$ is defined over $(\rho,+\infty)$ when $\sigma \geq 1$ while it is defined over $(\rho, \rho /(1-\sigma))$ when $\sigma \in(0,1)$. These restrictions allow to define lower and upper bounds for the discount factor $\rho$. Indeed, solving simultaneously $\mathcal{F}(\rho /(1-\sigma))=\mathcal{G}(\rho /(1-\sigma))$ and $\mathcal{F}(\rho)=\mathcal{G}(\rho)$ with respect to $\rho$ gives respectively

$$
\rho_{1}=(1-\sigma)\left(\frac{\beta_{21}}{\beta_{12}} \tilde{\beta}\right)^{1 /(1-\mu)}, \quad \rho_{2}=\left(\frac{\beta_{21}}{\beta_{11}} \tilde{\beta}\right)^{1 /(1-\mu)}
$$

Note that $\rho_{1}$ can be negative and the difference $\rho_{2}-\rho_{1}$ can be positive or negative.

From all these results, we now study the existence and uniqueness of a stationary balanced growth rate. Let us define

$$
\mu_{1}=\frac{\beta_{11}(\sigma-1)+\beta_{12}}{\sigma \beta_{11}}, \quad \mu_{2}=\frac{\beta_{11}(\sigma-1)+\beta_{12}}{\sigma \beta_{12}}
$$

i) When $\beta_{11}<\beta_{12}$, we get $\mu_{1}>\mu_{2}>1$. For any value of $\sigma>0, \mathcal{G}(r)$ is monotone increasing. Multiple solutions for equation (17) can only arise if $\mathcal{F}(r)$ is also increasing, i.e. when $\mu>0$. In this case, when $\rho=\rho^{*}, r^{*}$ is a tangency point between $\mathcal{F}(r)$ and $\mathcal{G}(r)$. Therefore multiplicity will hold in the following cases:

a) If $\sigma \geq 1$, we need to have $r^{*}>\rho^{*}$, i.e. $\mu \in\left(1, \mu_{1}\right)$. Recall that with $\mu>1, \mathcal{F}(r)$ is convex with $\lim _{r \rightarrow+\infty} \mathcal{F}^{\prime}(r)=+\infty$ and thus $\lim _{r \rightarrow+\infty}[\mathcal{F}(r)-$ $\mathcal{G}(r)]>0$. Since $\beta_{11}<\beta_{12}$, we have $\lim _{\rho \rightarrow+\infty} \mathcal{G}(0)=-\infty$. Then, when $\rho>\rho^{*}, \mathcal{F}(r)$ lies above $\mathcal{G}(r)$ and no intersection occur. When $\rho=\rho^{*}$, the tangency point $r^{*}$ is the unique solution of equation (17). When $\rho<\rho^{*}$, two solutions can occur provided $\rho$ is not too small. Indeed from the restriction $r>\rho$ we have to consider the solution of $\mathcal{F}(\rho)=\mathcal{G}(\rho)$ with respect to $\rho$. We get $\rho_{2}$ as defined by (30) and a condition for multiplicity is $\rho \in\left(\rho_{2}, \rho^{*}\right)$.

b) If $\sigma \in(0,1)$, we need to have $r^{*}>\rho^{*}$ and $r^{*}<\rho^{*} /(1-\sigma)$, i.e. $\mu \in\left(\mu_{2}, \mu_{1}\right)$. When $\rho>\rho^{*}, \mathcal{F}(r)$ lies above $\mathcal{G}(r)$ and no intersection occur. When $\rho=\rho^{*}$, the tangency point $r^{*}$ is the unique solution of equation (17). 
When $\rho<\rho^{*}$, from the restrictions $r>\rho$ and $r<\rho /(1-\sigma)$, we have to consider $\rho_{2}$ and the solution of $\mathcal{F}(\rho /(1-\sigma))=\mathcal{G}(\rho /(1-\sigma))$ with respect to $\rho$. We get $\rho_{1}$ as defined by (30). Now we define $\rho_{1}^{*}=\max \left\{0, \min \left\{\rho_{1}, \rho_{2}\right\}\right\}$ and $\rho_{2}^{*}=\max \left\{\rho_{1}, \rho_{2}\right\}$. Two solutions will occur provided $\rho \in\left(\rho_{2}^{*}, \rho^{*}\right)$.

Result $i$ ) is obtained from the restriction $\mu \in\left(\mu_{2}, \mu_{1}\right)$.

When $\beta_{11}>\beta_{12}, \mathcal{G}(r)$ is monotone increasing if $\sigma \geq\left(\beta_{11}-\beta_{12}\right) / \beta_{11} \equiv \hat{\sigma}$ and monotone decreasing if $\sigma<\hat{\sigma}$. Multiple solutions for equation (17) can only arise if $\mathcal{F}(r)$ and $\mathcal{G}(r)$ are both increasing or decreasing, and the tangency point is into the domain of definition.

ii) Consider first the case $\sigma \geq \hat{\sigma}$ in which $0<\mu_{1}<\mu_{2}<1$. Multiplicity then requires $\mu>0$.

a) If $\sigma \geq 1$, we need to have $r^{*}>\rho^{*}$, i.e. $\mu \in\left(\mu_{1}, 1\right)$. As in case $\left.i\right) a$ ), multiplicity is obtained under $\rho \in\left(\rho_{2}^{*}, \rho^{*}\right)$.

b) If $\sigma \in[\hat{\sigma}, 1)$, we need to have $r^{*}>\rho^{*}$ and $r^{*}<\rho^{*} /(1-\sigma)$, i.e. $\mu \in\left(\mu_{1}, \mu_{2}\right)$. As in case $\left.\left.i\right) b\right)$, multiplicity is obtained under $\rho \in\left(\rho_{2}^{*}, \rho^{*}\right)$.

Result ii) is obtained from the restriction $\mu \in\left(\mu_{1}, \mu_{2}\right)$.

iii) Consider finally the case $\sigma \in(0, \hat{\sigma}]$ in which $\mu_{2}<\mu_{1}<0$. Multiplicity then requires $\mu<0$. We also need to have $r^{*}>\rho^{*}$ and $r^{*}<\rho^{*} /(1-\sigma)$, i.e. $\mu \in\left(\mu_{2}, \mu_{1}\right)$. Since $\beta_{11}>\beta_{12}$, we have $\lim _{\rho \rightarrow+\infty} \mathcal{G}(0)=+\infty$. Then, when $\rho<\rho^{*}, \mathcal{F}(r)$ lies above $\mathcal{G}(r)$ and no intersection occurs. When $\rho=\rho^{*}$, the tangency point $r^{*}$ is the unique solution of equation (17). When $\rho>\rho^{*}$, two solutions can occur provided $\rho$ is not too big. From the restrictions $r>\rho$ and $r<\rho /(1-\sigma)$, we have to consider $\rho_{1}$ and $\rho_{2}$ as defined by (30). Two solutions will occur provided $\rho \in\left(\rho^{*}, \rho_{1}^{*}\right)$.

\subsection{Proof of Corollary 1}

i) Assume that $b_{1}=b_{2}=b>0$. We derive from (28) that $\mu>1$. It follows therefore from Theorem 1 that two stationary BGPs exist if $\beta_{11}<\beta_{12}$, $\mu \in\left(\mu_{2}, \mu_{1}\right) \subset(1,+\infty)$ and $\rho \in\left(\rho_{2}^{*}, \rho^{*}\right)$.

ii) Assume that $b_{1}=0$ and $b_{2} \in(0,1)$. We derive from (28) that $\mu>1$ and the same conclusions as in case i) hold.

iii) Assume that $b_{1} \in(0,1), b_{2}=0$ and $\beta_{22}=0$. We derive $\mu=\left(1+\beta_{12}\right) / b_{1}>$ 1. It follows therefore from Theorem 1 that two stationary BGPs exist if 
$\mu \in\left(\mu_{2}, \mu_{1}\right) \subset(1,+\infty)$ and $\rho \in\left(\rho_{2}^{*}, \rho^{*}\right)$.

\subsection{Proof of Theorem 2}

i) Consider the case $\sigma \geq 1$. Recall that the domain of definition of $r$ is $(\rho,+\infty)$. For any value of $\sigma \geq 1, \mathcal{G}(r)$ is monotone increasing. We will discuss the different configurations depending on the sign of $\beta_{11}-\beta_{12}$. Note that for any sign of this capital intensity difference, $\mathcal{F}(r)$ is non increasing when $\mu \leq 0$. It follows that if $\mu \leq 0$, there exists a unique solution for equation (17) provided $\mathcal{F}(\rho)>\mathcal{G}(\rho)$, i.e. $\rho<\rho_{2}^{*}=\rho_{2}$, with $\rho_{2}$ as defined by (30).

a) Let $\beta_{11}<\beta_{12}$. When $\mu \in(0,1], \mathcal{F}(r)$ is increasing and concave with $\lim _{r \rightarrow+\infty} \mathcal{F}^{\prime}(r)=0$. It follows that $\lim _{r \rightarrow+\infty}[\mathcal{F}(r)-\mathcal{G}(r)]<0$. Therefore if $\mu \leq 1$, there exists a unique solution for equation (17) provided $\mathcal{F}(\rho)>\mathcal{G}(\rho)$, i.e. $\rho<\rho_{2}^{*}$. If on the contrary $\mu>1, r^{*}$ is a tangency point between $\mathcal{F}(r)$ and $\mathcal{G}(r)$ when $\rho=\rho^{*}$. The interiority condition is $r^{*}>\rho^{*}$, i.e. $\mu<\mu_{1}$. If this inequality is satisfied, we know from Theorem 1 that existence and uniqueness will hold as soon as $\rho<\rho_{2}^{*}$. If the interiority condition does not hold, i.e. $\mu \geq \mu_{1}$, note that $\mathcal{F}(r)$ is increasing and convex with $\lim _{r \rightarrow+\infty}[\mathcal{F}(r)-\mathcal{G}(r)]>0$. Then there exists a unique solution for equation (17) provided $\mathcal{F}(\rho)<\mathcal{G}(\rho)$. Since $\mu>1$, this gives again the condition $\rho<\rho_{2}^{*}$.

b) Let $\beta_{11}>\beta_{12}$. When $\mu \in(0,1), r^{*}$ is a tangency point between $\mathcal{F}(r)$ and $\mathcal{G}(r)$ when $\rho=\rho^{*}$ provided the interiority condition $r^{*}>\rho^{*}$ is satisfied, i.e. $1>\mu>\mu_{1}$. In this case, Theorem 1 implies that existence and uniqueness will hold as soon as $\rho<\rho_{2}^{*}$. If the interiority condition does not hold, i.e. $\mu \leq \mu_{1}, \mathcal{F}(r)$ is concave with $\lim _{r \rightarrow+\infty}[\mathcal{F}(r)-\mathcal{G}(r)]<0$. Existence and uniquenes hold when $\mathcal{F}(\rho)>\mathcal{G}(\rho)$, i.e. $\rho<\rho_{2}^{*}$. Finally if $\mu>1, \mathcal{F}(r)$ is convex with $\lim _{r \rightarrow+\infty}[\mathcal{F}(r)-\mathcal{G}(r)]>0$. There exists a unique stationary balanced growth rate if $\mathcal{F}(\rho)<\mathcal{G}(\rho)$. Since $\mu \geq 1$, this gives again $\rho<\rho_{2}^{*}$.

ii) We have now to consider the case $\sigma \in(0,1)$. Recall that the domain of definition of $r$ is $(\rho, \rho /(1-\sigma))$. If $\beta_{11}<\beta_{12}, \mathcal{G}(r)$ is monotone increasing 
for any $\sigma \in(0,1)$. The same results as in the previous configurations $i$ - $-a$ ) hold except the fact that since $r \in(\rho, \rho /(1-\sigma))$, existence and uniqueness require $\mathcal{F}(\rho)>\mathcal{G}(\rho)$ and $\mathcal{F}(\rho /(1-\sigma))<\mathcal{G}(\rho /(1-\sigma))$. The restriction on the discount factor then becomes $\rho \in\left(\rho_{1}^{*}, \rho_{2}^{*}\right)$. If on the contrary $\beta_{11}>\beta_{12}$, $\mathcal{G}(r)$ is monotone increasing if $\sigma \geq \hat{\sigma}$ and monotone decreasing if $\sigma<\hat{\sigma}$. When $\sigma \geq \hat{\sigma}$, the same results as in the previous configurations $i$ )- $b$ ) hold with $\rho \in\left(\rho_{1}^{*}, \rho_{2}^{*}\right)$. We need therefore to study the case $\sigma \in(0, \hat{\sigma})$. When $\mu \leq 0, \mathcal{F}(r)$ is also non increasing. Then $r^{*}$ is a tangency point between $\mathcal{F}(r)$ and $\mathcal{G}(r)$ when $\rho=\rho^{*}$ provided the interiority conditions $r^{*}>\rho^{*}$ and $r^{*}<\rho^{*} /(1-\sigma)$ are satisfied, i.e. $\mu \in\left(\mu_{2}, \mu_{1}\right)$. In this case, Theorem 1 implies that existence and uniqueness will hold as soon as $\rho \in\left(\rho_{1}^{*}, \rho_{2}^{*}\right)$. If on the contrary the interiority conditions are not satisfied, we obtain the following conditions: if $\mu \leq \mu_{2}$, existence and uniqueness hold when $\mathcal{F}(\rho)<\mathcal{G}(\rho)$ and $\mathcal{F}(\rho /(1-\sigma))>\mathcal{G}(\rho /(1-\sigma))$. Since $\mu<0$ this gives again $\rho \in\left(\rho_{1}^{*}, \rho_{2}^{*}\right)$. On the contrary, if $0>\mu \geq \mu_{1}$, existence and uniqueness hold when $\mathcal{F}(\rho)>\mathcal{G}(\rho)$ and $\mathcal{F}(\rho /(1-\sigma))<\mathcal{G}(\rho /(1-\sigma))$, i.e. $\rho \in\left(\rho_{1}^{*}, \rho_{2}^{*}\right)$. Finally, if $\mu \geq 0 \mathcal{F}(r)$ is non decreasing and existence and uniqueness will hold when $\mathcal{F}(\rho)<\mathcal{G}(\rho)$ and $\mathcal{F}(\rho /(1-\sigma))>\mathcal{G}(\rho /(1-\sigma))$, i.e. $\rho \in\left(\rho_{1}^{*}, \rho_{2}^{*}\right)$.

\subsection{Proof of Proposition 2}

Let $k=k_{1} / k_{2}$. Linearizing the dynamical system (11)-(14) around a stationary BGP gives the Jacobian matrix

$$
J=\left(\begin{array}{cc}
J_{11} & J_{12} \\
J_{21}^{a}+J_{21}^{b}+J_{21}^{c} & J_{22}
\end{array}\right)
$$

with

$$
\begin{gathered}
J_{11}=\left(\begin{array}{cc}
r-\alpha_{11} & -\alpha_{21} \\
-\alpha_{12} & r-\alpha_{22}
\end{array}\right), \quad J_{12}=-r\left(\begin{array}{cc}
\theta_{11} \frac{p_{1}}{k_{1}} & \theta_{21} \frac{p_{1}}{k_{2}} \\
\theta_{12} \frac{p_{2}}{k_{1}} & \theta_{22} \frac{p_{2}}{k_{2}}
\end{array}\right), \\
J_{21}^{a}=\frac{1}{r}\left(\begin{array}{cc}
\frac{k_{1}}{p_{1}} \alpha_{11}^{2}+\frac{k_{2}}{p_{2}} \alpha_{12}^{2} & \frac{k_{1}}{p_{1}} \alpha_{11} \alpha_{21}+\frac{k_{2}}{p_{2}} \alpha_{12} \alpha_{22} \\
\frac{k_{1}}{p_{1}} \alpha_{11} \alpha_{21}+\frac{k_{2}}{p_{2}} \alpha_{12} \alpha_{22} & \frac{k_{1}}{p_{1}} \alpha_{21}^{2}+\frac{k_{2}}{p_{2}} \alpha_{22}^{2}
\end{array}\right)
\end{gathered}
$$




$$
J_{21}^{b}=-\left(\begin{array}{cc}
\frac{k_{1}}{p_{1}} \alpha_{11}+\frac{k_{2}}{p_{1}} \alpha_{12} & 0 \\
0 & \frac{k_{1}}{p_{2}} \alpha_{21}+\frac{k_{2}}{p_{2}} \alpha_{22}
\end{array}\right), J_{21}^{c}=\left(\begin{array}{cc}
\frac{1}{\sigma} p_{1}^{-\frac{1+\sigma}{\sigma}} & 0 \\
0 & 0
\end{array}\right)
$$

and

$$
J_{22}=\left(\begin{array}{cc}
\alpha_{11} \theta_{11}+\alpha_{12} \theta_{12} k^{-1}+\alpha_{11}-\gamma & \alpha_{12} \theta_{22}+\alpha_{11} \theta_{21} k+\alpha_{12} \\
\alpha_{21} \theta_{11}+\alpha_{22} \theta_{12} k^{-1}+\alpha_{21} & \alpha_{22} \theta_{22}+\alpha_{21} \theta_{21} k+\alpha_{22}-\gamma
\end{array}\right)
$$

For $i=0,1,2,3$, let $\kappa_{i}(\bar{r})$ be the sum of the principal minors of order $4-i$ of the Jacobian matrix (32) evaluated at some steady state $\bar{r}$. Some tedious computations available upon request allow to simplify the expressions of each $\kappa_{i}(\bar{r})$ and to provide the results of the Proposition with $\mathcal{T}(\bar{r})=\kappa_{1}(\bar{r})$, $\mathcal{S}(\bar{r})=\kappa_{2}(\bar{r}), \mathcal{D}(\bar{r})=\kappa_{3}(\bar{r})$ and $\Sigma(\bar{r})=\kappa_{4}(\bar{r})$.

\subsection{Proof of Lemma 3}

The tangency point $r^{*}$ and the corresponding value $\rho^{*}$ are defined as the solutions of the following system

$$
\mathcal{F}\left(r^{*}\right)=\mathcal{G}\left(r^{*}\right), \quad \mathcal{F}^{\prime}\left(r^{*}\right) \mathcal{G}^{\prime}\left(r^{*}\right)
$$

Since $\mathcal{G}(r)$ is linear and $\mathcal{F}(r)$ is parabolic we can write

$$
\mathcal{F}\left(r^{*}\right)=r \mathcal{G}^{\prime}\left(r^{*}\right)+\rho\left(\beta_{11}-\beta_{12}\right), \quad \mathcal{F}^{\prime}\left(r^{*}\right)=\mu \frac{\mathcal{F}\left(r^{*}\right)}{r^{*}}=\mathcal{G}^{\prime}\left(r^{*}\right)
$$

It follows that $\mu \mathcal{G}\left(r^{*}\right)=r^{*} \mathcal{G}^{\prime}\left(r^{*}\right)$. Consider now the term between brackets into the expression of $\mathcal{D}(r)$ given in Proposition 2. We have

$$
\mathcal{G}\left(r^{*}\right)(\mu-1)+\rho\left(\beta_{11}-\beta_{12}\right)=r \mathcal{G}^{\prime}\left(r^{*}\right)-\mathcal{G}\left(r^{*}\right)+\rho\left(\beta_{11}-\beta_{12}\right)=0
$$

Moreover, for any $\bar{r} \neq r^{*}$, we have

$$
\mathcal{G}(\bar{r})(\mu-1)+\rho\left(\beta_{11}-\beta_{12}\right)=\bar{r}\left(\mathcal{F}^{\prime}(\bar{r})-\mathcal{G}^{\prime}(\bar{r})\right)
$$

Under Assumptions 1-2, $\left[\bar{r}-\gamma\left(\beta_{11}-\beta_{12}\right)\right]>0$ and the result follows.

\subsection{Proof of Theorem 3}

Let $\sigma=1$.

1 - We start by the case $\beta_{11}<\beta_{12}$. Theorem 2 shows that when $\mu \leq 1$ and $\rho<\rho_{2}$, the unique steady state $\bar{r}$ is such that $\mathcal{F}^{\prime}(\bar{r})-\mathcal{G}^{\prime}(\bar{r})<0$ while $\bar{r}$ is such that $\mathcal{F}^{\prime}(\bar{r})-\mathcal{G}^{\prime}(\bar{r})>0$ when $\mu \geq \beta_{12} / \beta_{11}$ and $\rho<\rho_{2}$. Consider now 
Theorem 1 with $\mu \in\left(1, \beta_{12} / \beta_{11}\right)$ and $\rho<\rho^{*}$. When $\rho \in\left(\rho_{2}, \rho^{*}\right)$, denote the two steady states $\bar{r}_{1}, \bar{r}_{2}$ such that $\bar{r}_{1}<\bar{r}_{2}$. We then have $\mathcal{F}^{\prime}\left(\bar{r}_{1}\right)-\mathcal{G}^{\prime}\left(\bar{r}_{1}\right)<0$ and $\mathcal{F}^{\prime}\left(\bar{r}_{2}\right)-\mathcal{G}^{\prime}\left(\bar{r}_{2}\right)>0$. When $\rho \in\left(0, \rho_{2}\right]$, there is one unique steady state $\bar{r}_{2}$ such that $\mathcal{F}^{\prime}(\bar{r})-\mathcal{G}^{\prime}\left(\bar{r}_{2}\right)>0$. We then prove the three different cases:

i) When $\mu \leq 1$ and $\rho<\rho_{2}, \tilde{\mathcal{D}}(\bar{r})>0$. Moreover from equation (28) we necessarily have $b_{2}-b_{1}<0$. It follows that $\tilde{\mathcal{T}}(\bar{r})>0$ and thus $\tilde{\mathcal{T}}(\bar{r})+\bar{\delta}>0$ so that the steady state is locally unstable.

ii) When $\mu \in\left(1, \beta_{12} / \beta_{11}\right)$, we have $\tilde{\mathcal{D}}\left(\bar{r}_{1}\right)>0$ as long as $\rho \in\left(\rho_{2}, \rho^{*}\right)$ and local indeterminacy of order 2 holds if and only if $\tilde{\mathcal{T}}\left(\bar{r}_{1}\right)+\bar{\delta}_{1}<0$. On the contrary, for any $\rho \in\left(0, \rho^{*}\right), \tilde{\mathcal{D}}\left(\bar{r}_{2}\right)<0$ and $\left(\bar{r}_{2}, \bar{\gamma}_{2}\right)$ is saddle-point stable.

iii) Finally, when $\mu \geq \beta_{12} / \beta_{11}$ and $\rho<\rho_{2}, \tilde{\mathcal{D}}(\bar{r})<0$ and saddle-point stability holds.

2 - Consider now the case $\beta_{11}>\beta_{12}$. From Theorem 2 and Table 1, we derive that when $\mu \leq \beta_{12} / \beta_{11}$ and $\rho<\rho_{2}$, the unique steady state $\bar{r}$ is such that $\mathcal{F}^{\prime}(\bar{r})-\mathcal{G}^{\prime}(\bar{r})<0$ while $\bar{r}$ is such that $\mathcal{F}^{\prime}(\bar{r})-\mathcal{G}^{\prime}(\bar{r})>0$ when $\mu \geq 1$ and $\rho<\rho_{2}$. On the contrary Theorem 1 shows that when $\mu \in\left(\beta_{12} / \beta_{11}, 1\right)$ and $\rho \in\left(\rho_{2}, \rho^{*}\right)$, the two steady states $\bar{r}_{1}, \bar{r}_{2}$ are such that $\mathcal{F}^{\prime}\left(\bar{r}_{1}\right)-\mathcal{G}^{\prime}\left(\bar{r}_{1}\right)<0$ and $\mathcal{F}^{\prime}\left(\bar{r}_{2}\right)-\mathcal{G}^{\prime}\left(\bar{r}_{2}\right)>0$. When $\rho \in\left(0, \rho_{2}\right]$, the unique steady state $\bar{r}_{2}$ is such that $\mathcal{F}^{\prime}\left(\bar{r}_{2}\right)-\mathcal{G}^{\prime}\left(\bar{r}_{2}\right)>0$. We then prove the three different cases:

i) When $\mu \leq \beta_{12} / \beta_{11}$ and $\rho<\rho_{2}, \tilde{\mathcal{D}}(\bar{r})>0$ and from equation (28) we necessarily have $b_{12}+b_{21}<0$. It follows that $\tilde{\mathcal{T}}(\bar{r})>0$ and thus $\tilde{\mathcal{T}}(\bar{r})+\bar{\delta}>0$ so that the steady state is locally unstable.

ii) When $\mu \in\left(\beta_{12} / \beta_{11}, 1\right)$, we have $\tilde{\mathcal{D}}\left(\bar{r}_{1}\right)>0$ as long as $\rho \in\left(\rho_{2}, \rho^{*}\right)$ and for any $\rho \in\left(0, \rho^{*}\right), \tilde{\mathcal{D}}\left(\bar{r}_{2}\right)<0$. Since $b_{2}-b_{1}<0$, it follows that $\left(\bar{r}_{1}, \bar{\gamma}_{1}\right)$ is locally unstable while $\left(\bar{r}_{2}, \bar{\gamma}_{2}\right)$ is saddle-point stable.

iii) Finally, when $\mu \geq 1$ and $\rho<\rho_{2}, \tilde{\mathcal{D}}(\bar{r})<0$ and saddle-point stability holds.

\subsection{Proof of Theorem 4}

We will use the following Proposition from Barinci and Drugeon [3] which provides a set of useful criteria for assessing the local determinacy / indeterminacy of intertemporal equilibria in economies described by 3 -dimensional 
continuous-time dynamical systems.

Proposition 3. Consider an economy locally described by a characteristic polynomial of degree 3 such that

$$
g(\lambda)=\lambda^{3}-\mathcal{T} \lambda^{2}+\mathcal{S} \lambda-\mathcal{D}
$$

The dimension of the local stable manifold is as follows:

i) for $\mathcal{T}<0$, if $\mathcal{D}<0$ and $\mathcal{D}>\mathcal{S T}$, there are three eigenvalues with negative real parts;

ii) for $\mathcal{T}<0$ and $\mathcal{D}>0$ or for $\mathcal{T}>0, \mathcal{D}>0$ and $\mathcal{D}>\mathcal{S T}$, there are two eigenvalues with negative real parts;

iii) for $\mathcal{T}<0$, if $\mathcal{D}<0$ and $\mathcal{D}<\mathcal{S T}$ or, for $\mathcal{T}>0$, if $\mathcal{D}<0$, there is one eigenvalue with negative real part;

iv) for $\mathcal{T}>0$, if $\mathcal{D}>0$ and $\mathcal{D}<\mathcal{S} \mathcal{T}$, there is no eigenvalue with negative real part.

We now prove Theorem 4. Let $\beta_{11}<\beta_{12}$ and recall that

$$
\mathcal{D}(\bar{r})-\mathcal{S}(\bar{r}) \mathcal{T}(\bar{r})=-(\bar{\delta}+\tilde{\mathcal{T}}(\bar{r})) \tilde{\mathcal{D}}(\bar{r})-\mathcal{T}(\bar{r})[\bar{\delta}(\bar{\delta}+\tilde{\mathcal{T}}(\bar{r}))+\mathcal{Y}(\bar{r})]
$$

Depending on the value of $\sigma$ and $\mu$, the proof is derived from Theorems 1-2, Lemma 3, Propositions 2-3 and equation (33). We will only give a detailled argument for case 1 of Theorem 4 . Consider the bounds $\mu_{1}, \mu_{2}$ as defined by (28).

1 - Assume first that $\sigma>1$. From Proposition 2 we derive $\mathcal{Y}(\bar{r})>0$.

i) If $\mu \leq 1$ and $\rho<\rho_{2}^{*}$, then $\mathcal{D}(\bar{r})>0$. Moreover, we get from equation $(28)$ that $b_{2}-b_{1}$ is necessarily negative so that $\tilde{\mathcal{T}}(\bar{r})>0$. It follows that $\mathcal{T}(\bar{r})>0, \mathcal{S}(\bar{r})>0$ and, from equation $(33), \mathcal{D}(\bar{r})-\mathcal{S}(\bar{r}) \mathcal{T}(\bar{r})<0$. Proposition 3 implies that $(\bar{r}, \bar{\gamma})$ is locally unstable.

ii) Let $\mu \in\left(1, \mu_{1}\right)$ and consider Theorem 1 and Lemma 3. When $\rho \in$ $\left(\rho_{2}^{*}, \rho^{*}\right)$, the steady states $\left(\bar{r}_{1}, \bar{\gamma}_{1}\right)$ and $\left(\bar{r}_{2}, \bar{\gamma}_{2}\right)$ are such that: $\mathcal{D}\left(\bar{r}_{1}\right)>0$ and $\mathcal{D}\left(\bar{r}_{2}\right)<0$. Proposition 3 implies that $\left(\bar{r}_{1}, \bar{\gamma}_{1}\right)$ is locally indeterminate of order 2 if $\mathcal{T}\left(\bar{r}_{1}\right)<0$, or $\mathcal{T}\left(\bar{r}_{1}\right)>0$ and $\mathcal{D}\left(\bar{r}_{1}\right)-\mathcal{S}\left(\bar{r}_{1}\right) \mathcal{T}\left(\bar{r}_{1}\right)>0$, while $\left(\bar{r}_{2}, \bar{\gamma}_{2}\right)$ is locally indeterminate of order 3 if and only if $\mathcal{T}\left(\bar{r}_{2}\right)<0$ and $\mathcal{D}\left(\bar{r}_{2}\right)-\mathcal{S}\left(\bar{r}_{2}\right) \mathcal{T}\left(\bar{r}_{2}\right)>0$. When $\rho \leq \rho_{2}^{*}$, the unique remaining steady state $(\bar{r}, \bar{\gamma})$ is such that $\mathcal{D}(\bar{r})<0$. The result follows from Proposition 3. 
iii) If $\mu \geq \mu_{1}$ and $\rho<\rho_{2}^{*}$, then $\tilde{\mathcal{D}}(\bar{r})<0$. The result follows from Proposition 3.

2 - Assume now that $\sigma<1$. From Proposition 2 we derive $\mathcal{Y}(\bar{r})<0$.

i) If $\mu \leq 1$ and $\rho \in\left(\rho_{1}^{*}, \rho_{2}^{*}\right)$, we have $\tilde{\mathcal{T}}(\bar{r})>0$ and $\mathcal{D}(\bar{r})>0$.

ii) If $\mu \in\left(1, \mu_{2}\right)$ and $\rho \in\left(\rho_{1}^{*}, \rho_{2}^{*}\right)$, we have $\mathcal{D}(\bar{r})>0$.

iii) Let $\mu \in\left(\mu_{2}, \mu_{1}\right)$. When $\rho \in\left(\rho_{2}^{*}, \rho^{*}\right)$, the steady states $\left(\bar{r}_{1}, \bar{\gamma}_{1}\right)$ and $\left(\bar{r}_{2}, \bar{\gamma}_{2}\right)$ are such that: $\mathcal{D}\left(\bar{r}_{1}\right)>0$ and $\mathcal{D}\left(\bar{r}_{2}\right)<0$. When $\rho \in\left(\rho_{1}^{*}, \rho_{2}^{*}\right]$, consider the bounds $\rho_{1}$ and $\rho_{2}$ defined by equations (30). The unique remaining steady state $(\bar{r}, \bar{\gamma})$ is such that $\mathcal{D}(\bar{r})<0$ or $\mathcal{D}(\bar{r})>0$ depending on whether the difference $\rho_{2}-\rho_{1}$ is positive or negative. The result follows from the fact that $\rho_{2}-\rho_{1}>0$ if and only if $\sigma>1-\left(\beta_{12} / \beta_{11}\right)^{1 /(1-\mu)}$.

iv) If $\mu \geq \mu_{1}$ and $\rho \in\left(\rho_{1}^{*}, \rho_{2}^{*}\right)$, we have $\mathcal{D}(\bar{r})<0$.

\subsection{Proof of Theorem 5}

Let $\beta_{11}>\beta_{12}$. We proceed as in the proof of Theorem 4. Depending on the value of $\sigma$ and $\mu$, the results are derived from Theorems 1-2, Lemma 3, Propositions 2-3 and equation (33).

1 - Assume first that $\sigma>1$. From Proposition 2 we derive $\mathcal{Y}(\bar{r})<0$.

i) If $\mu \leq \mu_{1}$ and $\rho<\rho_{2}^{*}$, we have $\tilde{\mathcal{T}}(\bar{r})>0$ and $\mathcal{D}(\bar{r})>0$.

ii) If $\mu \in\left(\mu_{1}, 1\right)$, we have $\tilde{\mathcal{T}}(\bar{r})>0$. When $\rho \in\left(\rho_{2}^{*}, \rho^{*}\right)$, the steady states $\left(\bar{r}_{1}, \bar{\gamma}_{1}\right)$ and $\left(\bar{r}_{2}, \bar{\gamma}_{2}\right)$ are such that: $\mathcal{D}\left(\bar{r}_{1}\right)<0$ and $\mathcal{D}\left(\bar{r}_{2}\right)>0$. When $\rho \leq \rho_{2}^{*}$, the unique remaining steady state $(\bar{r}, \bar{\gamma})$ is such that $\mathcal{D}(\bar{r})>0$.

iii) If $\mu \geq 1$ and $\rho<\rho_{2}^{*}$, we have $\mathcal{D}(\bar{r})<0$.

2 - Assume now that $\sigma<1$. From Proposition 2 we derive $\mathcal{Y}(\bar{r})>0$.

i) If $\mu<1$, we necessarily have $\tilde{\mathcal{T}}(\bar{r})>0$. Therefore any steady state will be either saddle-point stable if $\mathcal{D}(\bar{r})<0$ or locally unstable if $\mathcal{D}(\bar{r})>0$ since in this case we have $\mathcal{S}(\bar{r})>0$ and $\mathcal{D}(\bar{r})-\mathcal{S}(\bar{r}) \mathcal{T}(\bar{r})<0$.

ii) If $\mu \geq 1$ and $\rho \in\left(\rho_{1}^{*}, \rho_{2}^{*}\right)$, we have $\mathcal{D}(\bar{r})<0$. 